\title{
Stokes Operators for Microhyperbolic Equations
}

\author{
By Keisuke UchiKoshi \\ Dedicated to Professor Hikosaburo Komatsu for his sixtieth birthday
}

\begin{abstract}
We consider microhyperbolic equations degenerated precisely on a hyperplane, and study the propagation of the singularities.
\end{abstract}

\section{Introduction}

In this paper we study the propagation of the singularity for some class of microhyperbolic operators, containing the case of non-involutive characteristics. As for microhyperbolic operators with non-involutive characteristics, there are many papers for the case of order two (c.f. $[1,2,4,5,6,10,11,12,14$, $15]$ ), and some papers for the case of higher orders (c.f. $[16,17,18]$ ). In these cases it is well-known that the propagation of the singularity is closely related to the classical theory for ordinary differential equations. In this paper, we generalize such a result, and give a general representation of the elementary solution: It is the composite of holomorphic microlocal operators and quantized contact transformations. As a natural consequence, we obtain the notion of Stokes operators. Such operators were previously known only for a very special case. Using these operators, we can study the branching of the singularity. Note that we do not assume any restrictive conditions for the lower order terms.

Let $P(x, D)$ be a microdifferential operator defined at $x^{*}=(0 ; 0, \cdots, 0$, $\sqrt{-1}) \in \mathbf{T}^{*} \mathbf{C}^{n}$ of order $m$, written in the form

$$
P(x, D)=D_{1}^{m}+\sum_{0 \leq j \leq m-1} P_{j}\left(x, D^{\prime}\right) D_{1}^{j}
$$

Here we have written $D^{\prime}=\left(D_{2}, \cdots, D_{n}\right)$ as usual. Sometimes we also write

1991 Mathematics Subject Classification. Primary 35L80; Secondary 35S30. 
as $D^{\prime \prime}=\left(D_{1}, \cdots, D_{n-1}\right)$ and $D^{\prime \prime \prime}=\left(D_{2}, \cdots, D_{n-1}\right)$. We assume that

(2) $\left\{\begin{array}{l}\text { there exist real holomorphic functions } \varphi_{1}\left(x, \xi^{\prime}\right), \cdots, \varphi_{m}\left(x, \xi^{\prime}\right) \\ \text { homogeneous in } \xi^{\prime} \text { of degree } 1 \text {, vanishing at } x^{*}, \text { and the principal } \\ \text { symbol of } P \text { is equal to }\left(\xi_{1}+\varphi_{1}\left(x, \xi^{\prime}\right)\right) \cdots\left(\xi_{1}+\varphi_{m}\left(x, \xi^{\prime}\right)\right) .\end{array}\right.$

We also assume that

(3) $\left\{\begin{array}{l}\text { if } i \neq j \text {, and }(x, \xi) \text { belongs to a small complex neighborhood } \\ \text { of } x^{*} \text {, then we have } \\ \varphi_{i}\left(x, \xi^{\prime}\right)=\varphi_{j}\left(x, \xi^{\prime}\right) \quad \Longleftrightarrow \quad x_{1}=0,\end{array}\right.$

and

(4) $\left\{\begin{array}{c}\text { for some } q_{j} \in \mathbf{N}=\{1,2,3, \cdots\} \text { and some } a_{j}\left(x, \xi^{\prime}\right) \in \mathcal{O}_{x^{*}} \text { we have } \\ \varphi_{j}\left(x, \xi^{\prime}\right)=x_{1}^{q_{j}} a_{j}\left(x, \xi^{\prime}\right), a_{j}\left(x^{*}\right) \neq 0(1 \leq j \leq m) \text {, and } \\ i \neq j \Longrightarrow \quad \Longrightarrow \quad\left(q_{i}, a_{i}\left(x^{*}\right)\right) \neq\left(q_{j}, a_{j}\left(x^{*}\right)\right) .\end{array}\right.$

In this paper we assume (1)-(4). It is easy to see that (4) is a sufficient condition of (3). Therefore in fact we assume (1), (2), and (4).

We denote by $\mathcal{C}($ resp. $\mathcal{E}$ ) the sheaf of microfunctions (resp. microdifferential operators). Let us consider the Cauchy problem

$$
\left\{\begin{array}{l}
P u=0, \\
D_{1}^{j-1} u\left(0, x^{\prime}\right)=v_{j}\left(x^{\prime}\right), \quad 1 \leq j \leq m,
\end{array}\right.
$$

where $u \in \mathcal{C}_{\mathbf{R}^{n}, x^{*}}$ and $v_{j} \in \mathcal{C}_{\mathbf{R}^{n-1}, x^{* \prime}}\left(x^{* \prime}=(0 ; 0, \cdots, 0, \sqrt{-1}) \in \mathbf{T}^{*} \mathbf{C}^{n-1}\right)$. We rewrite (5) in the following form:

$$
L \vec{u}=\overrightarrow{0}, \vec{u}\left(0, x^{\prime}\right)=\vec{v}\left(x^{\prime}\right) .
$$

Here $L(x, D)$ is an $m \times m$ matrix written in the form

$$
\begin{aligned}
L(x, D)= & D_{1} I_{m}+\left(\begin{array}{cccc}
\varphi_{1}\left(x, D^{\prime}\right), & -1, & & 0 \\
& \varphi_{2}\left(x, D^{\prime}\right), & \ddots & \\
& & \ddots & -1 \\
0 & & & \varphi_{m}\left(x, D^{\prime}\right)
\end{array}\right) \\
& +\left(\begin{array}{ccc} 
\\
& & \\
& & \\
b_{1}\left(x, D^{\prime}\right), & \cdots, & b_{m}\left(x, D^{\prime}\right)
\end{array}\right)
\end{aligned}
$$


with some $b_{j}\left(x, D^{\prime}\right) \in \mathcal{E}_{x^{*}}$ of order at most $m-j$. Here $\varphi_{j}\left(x, D^{\prime}\right)$ denotes the microdifferential operator whose complete symbol is equal to $\varphi_{j}\left(x, \xi^{\prime}\right)$, and $I_{m}$ denotes the unit matrix. Furthermore we have written

$$
\vec{u}(x)=\left(\begin{array}{c}
u_{1} \\
u_{2} \\
\vdots \\
u_{m}
\end{array}\right), \quad \vec{v}\left(x^{\prime}\right)=\left(\begin{array}{c}
v_{1} \\
v_{2} \\
\vdots \\
v_{m}
\end{array}\right)
$$

where

$$
\left\{\begin{array}{l}
u_{1}=u, \\
u_{j+1}=\left(D_{1}+\varphi_{j}\left(x, D^{\prime}\right)\right) u_{j}, \quad 1 \leq j \leq m-1
\end{array}\right.
$$

According to the general theory of microhyperbolic operators in [9] , there uniquely exists an elementary solution $\tilde{E}\left(x, y^{\prime}\right) \in$ $\left(\mathcal{C}_{\mathbf{R}^{2 n-1},\left(x^{*},-x^{* \prime}\right)}\right)^{m \times m}$ satisfying

$$
\left\{\begin{array}{l}
L(x, D) \tilde{E}\left(x, y^{\prime}\right)=O \\
\tilde{E}\left(0, x^{\prime}, y^{\prime}\right)=\operatorname{sp} \delta\left(x^{\prime}-y^{\prime}\right) I_{m}
\end{array}\right.
$$

Here $A^{m \times m}$ denotes the set of $m \times m$ matrices whose components belong to a set $A$. Note that we are regarding $x_{1}$ as a parameter, because $P$ and $L$ are of Kowalewski type. It is easy to see that

$$
E:\left(\mathcal{C}_{\mathbf{R}^{n}, x^{*}}\right)^{m} \ni \vec{u}(x) \longmapsto \int \tilde{E}\left(x, y^{\prime}\right) \vec{u}\left(x_{1}, y^{\prime}\right) d y^{\prime} \in\left(\mathcal{C}_{\mathbf{R}^{n}, x^{*}}\right)^{m}
$$

is well-defined, and the solution of (6) is $\vec{u}(x)=E \vec{v}\left(x^{\prime}\right)$.

We can calculate supp $\tilde{E}$ easily. Let $j \in\{1, \cdots, m\}$. Let $\psi_{j}(x, \eta)$ be the solution of

$$
\left\{\begin{array}{l}
\partial_{x_{1}} \psi_{j}(x, \eta)-\eta_{1}+\varphi_{j}\left(x, \partial_{x^{\prime}} \psi_{j}(x, \eta)\right)=0 \\
\psi_{j}\left(0, x^{\prime}, \eta\right)=\sum_{2 \leq k \leq m} x_{k} \eta_{k} .
\end{array}\right.
$$

If $y_{k}=\partial_{\eta_{k}} \psi_{j}(x, \eta), \xi_{k}=\partial_{x_{k}} \psi_{j}(x, \eta), 1 \leq k \leq m$, then $\kappa_{j}: \mathbf{T}^{*} \mathbf{C}^{n} \ni$ $(x, \xi) \longmapsto(y, \eta) \in \mathbf{T}^{*} \mathbf{C}^{n}$ is a real homogeneous symplectic transformation defined around $x^{*}$. Note that $y_{1}=x_{1}, \eta_{1}=\xi_{1}+\varphi_{j}\left(x, \xi^{\prime}\right), \kappa_{j}\left(x^{*}\right)=x^{*}$. It is easy to see that

$$
\begin{aligned}
& \operatorname{supp} \tilde{E}\left(x, y^{\prime}\right)=\bigcup_{1 \leq j \leq m}\left\{\left(x, y^{\prime} ; \xi, \eta^{\prime}\right) \infty ; \xi_{1}+\varphi_{j}\left(x, \xi^{\prime}\right)=0,\right. \\
& \left.y_{k}=\kappa_{j}^{*}\left(x_{k}\right), \eta_{k}=-\kappa_{j}^{*}\left(\xi_{k}\right), 2 \leq k \leq m\right\} .
\end{aligned}
$$

In the next section we shall give a complete expression of $\tilde{E}$. 


\section{Main Results}

We first discuss about a well-known result for quantized contact transformation. For each number $j \in\{1, \cdots, m\}$, let $\tilde{k}_{j}\left(x, y^{\prime}\right) \in \mathcal{C}_{\mathbf{R}^{2 n-1},\left(x^{*},-x^{* \prime}\right)}$ be the solution of

$$
\left\{\begin{array}{l}
\left(D_{1}+\varphi_{j}\left(x, D^{\prime}\right)\right) \tilde{k}_{j}\left(x, y^{\prime}\right)=0 \\
\tilde{k}_{j}\left(0, x^{\prime}, y^{\prime}\right)=\operatorname{sp} \delta\left(x^{\prime}-y^{\prime}\right)
\end{array}\right.
$$

We can define $k_{j}: \mathcal{C}_{\mathbf{R}^{n}, x^{*}} \ni u(x) \longmapsto \int \tilde{k}_{j}\left(x, y^{\prime}\right) u\left(x_{1}, y^{\prime}\right) d y^{\prime} \in \mathcal{C}_{\mathbf{R}^{n}, x^{*}}$, and it is a quantized contact transformation (i.e. Fourier integral operator) associated to $\kappa_{j}$. In fact we can calculate the complete symbol $k_{j}\left(x, \xi^{\prime}\right)$ of the operator $k_{j}$ in the form $\exp \left(\psi_{j}^{\prime}\left(x, \xi^{\prime}\right)\right) c_{j}\left(x, \xi^{\prime}\right)$ with some elliptic amplitude function $c_{j}$ of order at most 0 and the phase function $\psi_{j}^{\prime}\left(x, \xi^{\prime}\right)$, defined by

$$
\psi_{j}^{\prime}(x, \xi)\left(=\psi_{j}^{\prime}\left(x, \xi^{\prime}\right)\right)=\psi_{j}(x, \xi)-\sum_{1 \leq k \leq m} x_{k} \xi_{k}
$$

(See [7] ). Note that the kernel function $\tilde{k}_{j}$ is the microfunction defined by $(2 \pi \sqrt{-1})^{-n+1} \int \exp \left(\left(x^{\prime}-y^{\prime}\right) \cdot \xi^{\prime}\right) k_{j}\left(x, \xi^{\prime}\right) d \xi^{\prime}$.

Let $K:\left(\mathcal{C}_{\mathbf{R}^{n}, x^{*}}\right)^{m} \longrightarrow\left(\mathcal{C}_{\mathbf{R}^{n}, x^{*}}\right)^{m}$ be defined by

$$
K=\left(\begin{array}{ccc}
k_{1} & & 0 \\
& \ddots & \\
0 & & k_{m}
\end{array}\right) .
$$

Our first result is the following

ThEOREM 1. There exist a neighborhood $\omega^{\prime} \subset \sqrt{-1} \mathbf{S}^{*} \mathbf{R}^{n}$ of $x^{*} \infty, a$ neighborhood $\omega \subset \sqrt{-1} \mathbf{S}^{*} \mathbf{R}^{2 n-1}$ of $\left(x^{*},-x^{* \prime}\right) \infty$, and $m \times m$ invertible matrices

$$
Y_{ \pm}\left(x, D^{\prime}\right), Z_{ \pm}\left(x^{\prime}, D^{\prime}\right) \in\left(\mathcal{E}^{\mathbf{R}}\left(\omega_{ \pm}^{\prime}\right)\right)^{m \times m}
$$

such that $\tilde{E}$ is the kernel function of $Y_{ \pm} K Z_{ \pm}$on $\omega_{ \pm}$. Here $\mathcal{E}^{\mathrm{R}}$ denotes the sheaf of holomorphic microlocal operators, and

$$
\begin{aligned}
& \omega_{ \pm}=\left\{\left(x, y^{\prime} ; \xi, \eta^{\prime}\right) \infty \in \omega ; \pm x_{1}>0\right\} \\
& \omega_{ \pm}^{\prime}=\left\{(x, \xi) \infty \in \omega^{\prime} ; \pm x_{1}>0\right\} .
\end{aligned}
$$


Holomorphic microlocal operators are some class of analytic pseudodifferential operators. They are defined by [13], and [3] gave a symbol theory for them. They have microlocal property, i.e., if $A \in \mathcal{E}_{x^{*}}^{\mathbf{R}}, u \in \mathcal{C}_{\mathbf{R}^{n}, x^{*}}$, then we have $\operatorname{supp}(A u) \subset \operatorname{supp} u$.

Note that $Y_{ \pm}$(resp. $Z_{ \pm}$) are independent of $D_{1}$ (resp. $x_{1}$ and $D_{1}$ ). Therefore $Z_{ \pm}$are in fact defined for any $x_{1} . Z_{ \pm}$are the most important and we call them Stokes operators.

From (7) it follows that the singularity of the solution $\vec{u}$ of (6) propagates along the union of the bicharacteristic strips of $P$. Let us discuss about it in detail. Let $b_{j}$ be the bicharacteristic strip associated to $\xi_{1}+\varphi_{j}\left(x, \xi^{\prime}\right)$ through $x^{*} \infty$, and let $b_{ \pm, j}=\left\{(x, \xi) \infty \in b_{j} ; \pm x_{1}>0\right\}$. It is easy to see that

$$
\begin{aligned}
L(x, D) \vec{u}(x)=\overrightarrow{0} \quad & \Longleftrightarrow \quad \vec{u}(x)=E \vec{v}\left(x^{\prime}\right) \text { for some } \vec{v}\left(x^{\prime}\right) \\
& \Longleftrightarrow \quad \vec{u}(x)=E_{+} \vec{v}_{+}\left(x^{\prime}\right) \text { for some } \vec{v}_{+}\left(x^{\prime}\right) \\
& \Longleftrightarrow \quad \vec{u}(x)=E_{-} \vec{v}_{-}\left(x^{\prime}\right) \text { for some } \vec{v}_{-}\left(x^{\prime}\right),
\end{aligned}
$$

where $E_{ \pm}=Y_{ \pm} K=E\left(Z_{ \pm}\right)^{-1}$. Using the last statement of these equivalent conditions, the propagation in $\omega_{-}$is of trivial type. If $\vec{u}(x)={ }^{t}\left(u_{1}, \cdots, u_{m}\right)$, then we define $\operatorname{supp} \vec{u}=\bigcup_{1 \leq j \leq m} \operatorname{supp} u_{j}$. It follows that

$$
\begin{aligned}
\operatorname{supp}\left(E_{-} \vec{v}_{-}\left(x^{\prime}\right)\right) & =\operatorname{supp}\left(Y_{-} K \vec{v}_{-}\left(x^{\prime}\right)\right)=\operatorname{supp}\left(K \vec{v}_{-}\left(x^{\prime}\right)\right) \\
& =\operatorname{supp}\left(\begin{array}{c}
k_{1} v_{-, 1} \\
k_{2} v_{-, 2} \\
\vdots \\
k_{m} v_{-, m}
\end{array}\right)=\bigcup_{1 \leq j \leq m} \operatorname{supp}\left(k_{j} v_{-, j}\right)
\end{aligned}
$$

on $\omega_{-}$. Let $L \vec{u}=\overrightarrow{0}$. Since $\operatorname{supp}\left(k_{j} v_{-, j}\right)$ is contained in $\left\{\kappa_{j}(x, \xi) \in \omega_{-}\right.$; $\left.(x, \xi) \in \operatorname{supp} v_{-}\right\}$, we have, for instance,

$$
\begin{aligned}
\operatorname{supp} \vec{u} \cap \omega_{-}= & b_{-, 1} \\
\Longleftrightarrow & \text { the components of } \vec{v}_{-} \text {vanish except for } v_{-, 1} \\
& \quad \text { and } \operatorname{supp} v_{-, 1}=\left\{x^{* \prime} \infty\right\} .
\end{aligned}
$$

More generally $\operatorname{supp} \vec{u}$ contains $b_{-, j}$ if, and only if, $\operatorname{supp} v_{-, j}$ contains $\left\{x^{* \prime} \infty\right\}$. 
On the other hand, $E_{-}=Y_{+} K Z_{+}\left(Z_{-}\right)^{-1}$ on $\omega_{+}$. In this expression $E_{-}$ does not have such a diagonal property because of the additional factor $Z_{+-}=Z_{+}\left(Z_{-}\right)^{-1}$ composed from the right, and the situation in $\omega_{+}$is not so simple. In fact we have $\operatorname{supp}\left(E_{-} \vec{v}_{-}\left(x^{\prime}\right)\right)=\operatorname{supp}\left(Y_{+} K Z_{+-} \vec{v}_{-}\left(x^{\prime}\right)\right)=$ $\operatorname{supp}\left(K Z_{+-} \vec{v}_{-}\left(x^{\prime}\right)\right)$ on $\omega_{+}$. For instance assume the equivalent conditions in (8). As is well-known in the case $m=2$, the singularity may branch into different bicharacteristic strips on $\omega_{+}$. We denote the $(\mu, \nu)$-component of $Z_{+-}$by $Z_{+-,(\mu, \nu)}$. Then we have

$$
\begin{aligned}
& \operatorname{supp}\left(E_{-} \vec{v}_{-}\left(x^{\prime}\right)\right)=\operatorname{supp}\left(\begin{array}{cc}
k_{1} Z_{+-,(1,1)} & v_{-, 1} \\
k_{2} Z_{+-,(2,1)} & v_{-, 1} \\
\vdots & \\
k_{m} Z_{+-,(m, 1)} & v_{-, 1}
\end{array}\right) \\
& =\bigcup_{1 \leq j \leq m}\left\{\kappa_{j}(x, \xi) \in \omega_{-} ;(x, \xi) \in \operatorname{supp} Z_{+-,(j, 1)} v_{-, 1}\right\}
\end{aligned}
$$

on $\omega_{+}$.

More generally, $k_{j} Z_{+-,(i, j)}\left(x^{\prime}, D^{\prime}\right) k_{i}^{-1}$ denotes the operator transporting the singularity on $\left\{(x, \xi) \in \omega_{-} ; \xi_{1}+\varphi_{i}\left(x, \xi^{\prime}\right)=0\right\}$ into $\left\{(x, \xi) \in \omega_{+} ; \xi_{1}+\right.$ $\left.\varphi_{j}\left(x, \xi^{\prime}\right)=0\right\}$. We want to study how the singularity, which propagated along a simple bicharacteristic strip in the past, will branch in the future. For that purpose we need to calculate $Z_{+-}$. This is not so easy in general, but we have the following

THEOREm 2. Let $J$ be the set of those $j \in\{1, \cdots, m\}$ which satisfy $q_{j}=1$, and let $j_{0} \in J$. We assume that either

$$
\left\{\begin{array}{l}
\operatorname{Im} a_{j_{0}}\left(x^{*}\right)<0, \\
j \in J \backslash\left\{j_{o}\right\} \Longrightarrow \operatorname{Im} a_{j_{0}}\left(x^{*}\right)<\operatorname{Im} a_{j}\left(x^{*}\right)
\end{array}\right.
$$

or

$$
\left\{\begin{array}{l}
\operatorname{Im} a_{j_{0}}\left(x^{*}\right)>0, \\
j \in J \backslash\left\{j_{o}\right\} \Longrightarrow \operatorname{Im} a_{j_{0}}\left(x^{*}\right)>\operatorname{Im} a_{j}\left(x^{*}\right) .
\end{array}\right.
$$

Then $Z_{+-,\left(j_{0}, j_{0}\right)}\left(x^{\prime}, D^{\prime}\right)$ is invertible.

COROLLARY. Under the above assumption we have $\vec{u} \in \mathcal{C}_{\mathbf{R}^{n}, x^{*}} \backslash\{\overrightarrow{0}\}, L \vec{u}=\overrightarrow{0}, \operatorname{supp} \vec{u} \cap \omega_{-}=b_{-, j_{0}} \Longrightarrow \operatorname{supp} \vec{u} \cap \omega_{+} \supset b_{+, j_{0}}$. 
Example. If $m=4$ and

$$
\varphi_{1}=x_{1} \xi_{n}, \varphi_{2}=2 x_{1} \xi_{n}, \varphi_{3}=x_{1}^{2} \xi_{n}, \varphi_{4}=x_{1}^{3} \xi_{n}
$$

then $J=\{1,2\}$. If $j_{0}=2$, we have (10), and the above Corollary is true for this number.

The assumption (9) (resp. (10)) means that the characteristic root $\varphi_{j_{0}}\left(x, \xi^{\prime}\right)$ is the most negative (resp. positive), and for such a dominant root, at least some part of the singularity penetrates (from the past to the future) along the corresponding strip. We do not know whether the assumption (9) or (10) is indispensable or not. To the contrary, if $j_{0} \notin J$, the above conclusion is not always true. See [10] for examples of this fact.

\section{Transformation by Holomorphic Microlocal Operators}

Let $q=\max \left(q_{1}, \cdots, q_{m}\right)$. Let $C>1, \theta \in[0,2 \pi], i \in \mathbf{Z}_{+}=\{0,1,2, \cdots\}$. We define

$$
\begin{aligned}
& \Omega(C)=\left\{\left(x, \xi^{\prime}\right) \in \mathbf{C}^{n} \times \mathbf{C}^{n-1} ; C\left|x_{1}\right|<1, C\left|x^{\prime}\right|<1,\right. \\
& \left.\quad C\left|\xi^{\prime \prime \prime}\right|<\operatorname{Im} \xi_{n}, C\left|\operatorname{Re} \xi_{n}\right|<\operatorname{Im} \xi_{n}, C^{4 m q}<\operatorname{Im} \xi_{n}\right\}, \\
& \Omega_{i}(C)=\left\{\left(x, \xi^{\prime}\right) \in \Omega(C) ; C^{4 m q}(i+1)<\operatorname{Im} \xi_{n}\right\}, \\
& \Omega^{\theta}(C)=\left\{\left(x, \xi^{\prime}\right) \in \Omega(C) ; x_{1} \neq 0, C\left|\arg x_{1}-\theta\right|<1\right\}, \\
& \Omega_{i}^{\prime \theta}(C)=\left\{\left(x, \xi^{\prime}\right) \in \Omega_{i}(C) ;\left|x_{1}\right|>C\left(\operatorname{Im} \xi_{n}\right)^{-1 / m q}, C\left|\arg x_{1}-\theta\right|<1\right\}, \\
& \Omega^{\prime \prime}{ }_{i}(C)=\left\{\left(x, \xi^{\prime}\right) \in \Omega_{i}(C) ;\left|x_{1}\right|<C^{-1}\left(\operatorname{Im} \xi_{n}\right)^{-1 / 2 m q}\right\}, \\
& \Omega_{i}^{\theta}(C)=\Omega^{\prime \theta}(C) \cup \Omega^{\prime \prime}{ }_{i}(C) .
\end{aligned}
$$

Let $M(x, D)=D_{1} I_{m}+\bar{M}\left(x, D^{\prime}\right) \in\left(\mathcal{E}_{x^{*}}\right)^{m \times m}$, where

$$
\bar{M}\left(x, D^{\prime}\right)=\left(\begin{array}{ccc}
\varphi_{1}\left(x, D^{\prime}\right), & & 0 \\
0 & \ddots & \\
0 & & \varphi_{m}\left(x, D^{\prime}\right)
\end{array}\right) .
$$

Then we have the following 
Proposition 1. Let $\theta \in[0,2 \pi]$. There exist $Y^{ \pm, \theta}\left(x, D^{\prime}\right) \in$ $\left(\mathcal{E}^{\mathbf{R}}\left(\Omega^{\theta}(C)\right)\right)^{m \times m}$ such that

$$
\left\{\begin{array}{l}
L(x, D) Y^{+, \theta}\left(x, D^{\prime}\right)=Y^{+, \theta}\left(x, D^{\prime}\right) M(x, D), \\
Y^{+, \theta}\left(x, D^{\prime}\right) Y^{-, \theta}\left(x, D^{\prime}\right)=Y^{-, \theta}\left(x, D^{\prime}\right) Y^{+, \theta}\left(x, D^{\prime}\right)=\mathrm{Id}
\end{array}\right.
$$

on $\Omega^{\theta}(C)$.

REMARK. In Theorem 1, the inferior sign \pm of $Y_{ \pm}\left(x, D^{\prime}\right)$ means that this is an operator defined on $\omega_{ \pm}$. In Proposition 1 the superior sign of $Y^{ \pm, \theta}\left(x, D^{\prime}\right)$ stands for a different meaning. In this case $Y^{+, \theta}\left(x, D^{\prime}\right)$ is some operator, and $Y^{-, \theta}\left(x, D^{\prime}\right)$ is its inverse. We shall always use such a distinction.

To prove Proposition 1, we need to prepare a symbol theory for holomorphic microlocal operators containing $x_{1}$ as a parameter. It is the same as [3], but we resume the result for the sake of reader's convenience. We denote by $\mathcal{S}\left(\Omega^{\theta}(C)\right)$ the set of formal series $f=\sum_{i \in \mathbf{Z}_{+}} f_{i}\left(x, \xi^{\prime}\right)$ satisfying

(i) $f_{i} \in \mathcal{O}\left(\Omega_{i}^{\theta}(C)\right), i \in \mathbf{Z}_{+}$,

(ii) there exists some $R \in(0,1)$, and for any $\varepsilon>0$ there exists some $C_{\varepsilon}>0$ such that

$$
\left|f_{i}\left(x, \xi^{\prime}\right)\right| \leq C_{\varepsilon} R^{i} \exp \left(\varepsilon \operatorname{Im} \xi_{n}\right) \quad \text { on } \Omega_{i}^{\theta}(C), \quad \text { for each } i .
$$

To emphasize that this is a formal series, we write as $f=\sum_{i \in \mathbf{Z}_{+}}{ }^{*} f_{i}$. If $f=\sum_{i}^{*} f_{i}$, we define a formal series $f^{\sharp}=\sum_{i \in \mathbf{Z}_{+}}{ }^{*} f_{i}^{\sharp}$ by $f_{i}^{\sharp}=\sum_{0 \leq j \leq i} f_{j}$, and $\mathcal{N}\left(\Omega^{\theta}(C)\right)$ by

$$
\mathcal{N}\left(\Omega^{\theta}(C)\right)=\left\{f \in \mathcal{S}\left(\Omega^{\theta}(C)\right) ; f^{\sharp} \in \mathcal{S}\left(\Omega^{\theta}(C)\right)\right\} .
$$

We identify a function $f_{0}$ with $f_{0}+0+0+\cdots \in \mathcal{S}\left(\Omega^{\theta}(C)\right)$, if it satisfies (i) and (ii) for $i=0$.

Finally we define

$$
\mathcal{S}^{\theta}=\varliminf_{C>0} \mathcal{S}\left(\Omega^{\theta}(C)\right), \quad \mathcal{N}^{\theta}=\varliminf_{C>0} \mathcal{N}\left(\Omega^{\theta}(C)\right) .
$$


REMARK. (i) We write $\sum^{*} f_{i}=\sum^{*} g_{i}$ if, and only if, $f_{i}=g_{i}$ for any $i$. This does not simply mean that the sums of these two series are the same.

(ii) Let $\sum^{*} f_{i} \in \mathcal{S}\left(\Omega^{\theta}(C)\right)$, let $g_{0}=0$ and $g_{i}=f_{i-1}$ for $i \geq 1$. Then we have $\sum^{*}\left(f_{i}-g_{i}\right) \in \mathcal{N}\left(\Omega^{\theta}(C)\right)$.

(iii) A function $f_{0}$ belongs to $\mathcal{N}\left(\Omega^{\theta}(C)\right)$ if, and only if, it is exponentially decreasing.

(iv) If $f \in \mathcal{S}^{\theta}$, then $f\left(0, x^{\prime}, \xi^{\prime}\right) \in \mathcal{S}^{\theta}$ is well-defined.

(v) For a formal series $f=\sum^{*} f_{i}$ we define $\partial_{x_{1}} f=\sum^{*} \partial_{x_{1}} f_{i}$.

Let $f=\sum^{*} f_{i}\left(x, \xi^{\prime}\right) \in \mathcal{S}\left(\Omega^{\theta}(C)\right)$. We define $\mathcal{F}(f)\left(x, y^{\prime}\right)$ by

$$
\mathcal{F}(f)\left(x, y^{\prime}\right)=(2 \pi \sqrt{-1})^{n-1} \sum_{i} \int_{\Delta_{i}} e^{\xi^{\prime} \cdot\left(x^{\prime}-y^{\prime}\right)} f_{i}\left(x, \xi^{\prime}\right) d \xi^{\prime}
$$

where $\Delta_{i}=\left\{\xi^{\prime} \in \sqrt{-1} \mathbf{R}^{n-1} ; C\left|\xi^{\prime \prime \prime}\right|<\operatorname{Im} \xi_{n}, C(i+1)<\operatorname{Im} \xi_{n}\right\}$. Then we have the following

LEMMA 1. Let $f=\sum^{*} f_{i}\left(x, \xi^{\prime}\right) \in \mathcal{S}\left(\Omega^{\theta}(C)\right)$. $\mathcal{F}(f)$ is holomorphic on $\left\{\left(x, y^{\prime}\right) \in \mathbf{C}^{n} \times \mathbf{C}^{n-1} ; C^{\prime}\left|\left(x, y^{\prime}\right)\right|<1, x_{1} \neq 0, C^{\prime}\left|\arg x_{1}-\theta\right|<1, \operatorname{Re}\left(\xi^{\prime}\right.\right.$. $\left.\left(x^{\prime}-y^{\prime}\right)\right)<0$ for any $\left.\xi^{\prime} \in \Delta_{0}\right\}$ for $0<C<<C^{\prime}$, and it defines the kernel function of a holomorphic microlocal operator on $\Omega^{\theta}\left(C^{\prime}\right)$ (We denote this operator by $\left.\mathcal{M}(f)\left(x, D^{\prime}\right)\right)$. If $f \in \mathcal{N}^{\theta}$, then $\mathcal{M}(f)=0$.

Lemma 2. Let $f=\sum^{*} f_{i}\left(x, \xi^{\prime}\right), g=\sum^{*} g_{i}\left(x, \xi^{\prime}\right) \in \mathcal{S}^{\theta}$. We can define $f \circ g \in \mathcal{S}^{\theta}$ by

$$
(f \circ g)_{i}=\sum_{j+k+\left|\alpha^{\prime}\right|=i} \frac{1}{\alpha^{\prime} !} \partial_{\xi^{\prime}}^{\alpha^{\prime}} f_{j} \partial_{x^{\prime}}^{\alpha^{\prime}} g_{k},
$$

and we have

$$
\mathcal{M}(f)\left(x, D^{\prime}\right) \mathcal{M}(g)\left(x, D^{\prime}\right)=\mathcal{M}(f \circ g)\left(x, D^{\prime}\right)
$$

If $A(x, D)=\sum_{\alpha} A_{\alpha} D^{\alpha} \in \mathcal{E}_{x^{*}}$, then we define $\sigma_{i}(A)(x, \xi)=\sum_{|\alpha|=i} A_{\alpha} \xi^{\alpha}$. The complete symbols of $\bar{L}\left(x, D^{\prime}\right)=L(x, D)-D_{1} I_{m}$ and $\bar{M}\left(x, D^{\prime}\right)$ are 
graded as follows:

$$
\begin{aligned}
& \bar{L}_{i}=\delta_{i, 0}\left(\begin{array}{cccc}
\varphi_{1}\left(x, \xi^{\prime}\right), & -1, & & 0 \\
& \varphi_{2}\left(x, \xi^{\prime}\right), & \ddots & \\
& & \ddots & -1 \\
0 & & & \varphi_{m}\left(x, \xi^{\prime}\right)
\end{array}\right) \\
& +\left(\begin{array}{rrr} 
& & \\
& 0 & \\
\sigma_{m-1-i}\left(b_{1}\right)\left(x, \xi^{\prime}\right), & \cdots & \sigma_{-i}\left(b_{m}\right)\left(x, \xi^{\prime}\right)
\end{array}\right),
\end{aligned}
$$

and

$$
\bar{M}_{i}=\delta_{i, 0} \bar{M}\left(x, \xi^{\prime}\right)
$$

Note that $\bar{L}_{i}$ may not be homogeneous in $\xi^{\prime}$ of degree $i$. Now Proposition 1 is a corollary of the following

Proposition 2. Let $\theta \in[0,2 \pi]$. Then there exist $Y^{ \pm, \theta}\left(x, \xi^{\prime}\right)\left(x, \xi^{\prime}\right) \in$ $\left(\mathcal{S}^{\theta}\right)^{m \times m}$ such that

$$
\left\{\begin{array}{l}
\partial_{x_{1}} Y^{+, \theta}\left(x, \xi^{\prime}\right)+\bar{L}\left(x, \xi^{\prime}\right) \circ Y^{+, \theta}\left(x, \xi^{\prime}\right)-Y^{+, \theta}\left(x, \xi^{\prime}\right) \circ \bar{M}\left(x, \xi^{\prime}\right)=O, \\
Y^{ \pm, \theta}\left(x, \xi^{\prime}\right) \circ Y^{\mp, \theta}\left(x, \xi^{\prime}\right)=I_{m} .
\end{array}\right.
$$

In the next section we shall give of the proof of Proposition 2.

Now we can prove Theorem 1 as follows. Let $0 \leq \theta_{1}<\theta_{2}<\cdots<\theta_{\ell} \leq 2 \pi$ be such that, Proposition 1 is true for each $\theta_{j}$ with $C=B_{j}$ respectively, and $[0,2 \pi] \subset \bigcup_{1 \leq j \leq \ell}\left(\theta_{j}-B_{j}^{-1}, \theta_{j}+B_{j}^{-1}\right)$. Note that we can always choose such numbers. Let $K=K\left(x, \xi^{\prime}\right)$ be the complete symbol of the Fourier integral operator $K$, and let $Z^{\theta_{j}}\left(x^{\prime}, \xi^{\prime}\right)=Y^{-, \theta_{j}}\left(0, x^{\prime}, \xi^{\prime}\right)$ for each $\theta_{j}$. We may assume that $\partial_{x_{1}} K+\bar{M} \circ K=0, K\left(0, x^{\prime}, \xi^{\prime}\right)=I_{m}$ without any modulo classes. Then we have $\left(Y^{+, \theta_{i}} \circ K \circ Z^{\theta_{i}}\left(x^{\prime}, \xi^{\prime}\right)\right)_{k}=\left(Y^{+, \theta_{j}} \circ K \circ Z^{\theta_{j}}\left(x^{\prime}, \xi^{\prime}\right)\right)_{k}$ on $\Omega_{k}^{\theta_{i}}\left(B_{i}\right) \cap \Omega_{k}^{\theta_{j}}\left(B_{j}\right)$. In fact, we have

$$
\partial_{x_{1}}\left(Y^{+, \theta_{i}} \circ K \circ Z^{\theta_{i}}\left(x^{\prime}, \xi^{\prime}\right)\right)+\bar{L} \circ\left(Y^{+, \theta_{i}} \circ K \circ Z^{\theta_{i}}\left(x^{\prime}, \xi^{\prime}\right)\right)
$$




$$
\begin{aligned}
& =\left(\partial_{x_{1}} Y^{+, \theta_{i}}+\bar{L} \circ Y^{+, \theta_{i}}\right) \circ K \circ Z^{\theta_{i}}\left(x^{\prime}, \xi^{\prime}\right)+Y^{+, \theta_{i}} \circ \partial_{x_{1}}\left(K \circ Z^{\theta_{i}}\left(x^{\prime}, \xi^{\prime}\right)\right) \\
& =Y^{+, \theta_{i}} \circ \bar{M} \circ K \circ Z^{\theta_{i}}\left(x^{\prime}, \xi^{\prime}\right)+Y^{+, \theta_{i}} \circ \partial_{x_{1}}\left(K \circ Z^{\theta_{i}}\left(x^{\prime}, \xi^{\prime}\right)\right) \\
& =Y^{+, \theta_{i}} \circ\left(\partial_{x_{1}} K+\bar{M} \circ K\right) \circ Z^{\theta_{i}}\left(x^{\prime}, \xi^{\prime}\right)=O,
\end{aligned}
$$

and

$$
\left[Y^{+, \theta_{i}} \circ K \circ Z^{\theta_{i}}\left(x^{\prime}, \xi^{\prime}\right)\right]_{x_{1}=0}=Y^{+, \theta_{i}}\left(0, x^{\prime}, \xi^{\prime}\right) \circ I_{m} \circ Y^{-, \theta_{i}}\left(0, x^{\prime}, \xi^{\prime}\right)=I_{m}
$$

It follows that

$$
\left\{\begin{array}{l}
\partial_{x_{1}}\left(Y^{+, \theta_{i}} \circ K \circ Z^{\theta_{i}}\left(x^{\prime}, \xi^{\prime}\right)\right)+\bar{L} \circ\left(Y^{+, \theta_{i}} \circ K \circ Z^{\theta_{i}}\left(x^{\prime}, \xi^{\prime}\right)\right)=O, \\
{\left[Y^{+, \theta_{i}} \circ K \circ Z^{\theta_{i}}\left(x^{\prime}, \xi^{\prime}\right)\right]_{x_{1}=0}=I_{m}}
\end{array}\right.
$$

for each $\theta_{i}$. It is easy to see that such a symbol is unique, and we obtain

$$
\left(Y^{+, \theta_{i}} \circ K \circ Z^{\theta_{i}}\left(x^{\prime}, \xi^{\prime}\right)\right)_{k}=\left(Y^{+, \theta_{j}} \circ K \circ Z^{\theta_{j}}\left(x^{\prime}, \xi^{\prime}\right)\right)_{k}
$$

on $\Omega_{k}^{\theta_{i}}\left(B_{i}\right) \cap \Omega_{k}^{\theta_{j}}\left(B_{j}\right)$. Therefore $\left\{\left(Y^{+, \theta_{i}} \circ K\left(x, \xi^{\prime}\right) \circ Z^{\theta_{i}}\left(x^{\prime}, \xi^{\prime}\right)\right)_{k} ; 1 \leq i \leq \ell\right\}$ defines a holomorphic function on $\bigcup_{1 \leq j \leq \ell} \Omega_{k}^{\theta_{j}}\left(B_{j}\right)$. Increasing $C>0$ if necessary, the last set contains $\Omega_{k}(C)$. Since $0 \in\left(\theta_{j}-B_{j}^{-1}, \theta_{j}+B_{j}^{-1}\right)$ and $\pi \in\left(\theta_{k}-B_{k}^{-1}, \theta_{k}+B_{k}^{-1}\right)$ for some $j$ and $k$, we obtain Theorem 1 by setting

$$
\begin{array}{ll}
Y_{+}\left(x, \xi^{\prime}\right)=Y^{+, \theta_{j}}\left(x, \xi^{\prime}\right), & Z_{+}\left(x, \xi^{\prime}\right)=Y^{-, \theta_{j}}\left(0, x^{\prime}, \xi^{\prime}\right), \\
Y_{-}\left(x, \xi^{\prime}\right)=Y^{+, \theta_{k}}\left(x, \xi^{\prime}\right), & Z_{-}\left(x, \xi^{\prime}\right)=Y^{-, \theta_{k}}\left(0, x^{\prime}, \xi^{\prime}\right) .
\end{array}
$$

\section{Miscellanea}

In this section we give some lemmas necessary for the proof of Proposition 2. Without loss of generality, we may assume that the characteristic roots are aligned in such a way that

$$
q_{1} \leq q_{2} \leq \cdots \leq q_{m}
$$

and if $q_{i}=q_{j}, i<j$, then we have either

$$
\operatorname{Im} a_{i}\left(x^{*}\right) \cdot \operatorname{Im} a_{j}\left(x^{*}\right)<0
$$


or

$$
\operatorname{Im} a_{i}\left(x^{*}\right) \cdot \operatorname{Im} a_{j}\left(x^{*}\right)>0,\left|\operatorname{Im} a_{i}\left(x^{*}\right)\right|>\left|\operatorname{Im} a_{j}\left(x^{*}\right)\right| .
$$

Let $q=\max _{1 \leq j \leq m} q_{j}$. Therefore $q=q_{m}$ under the above assumption. Considering an arbitrary $\theta$ we omit the index $\theta$ of $Y_{i}^{+, \theta}$ for the moment. Let us solve

$$
\partial_{x_{1}} Y^{+}+\bar{L} \circ Y^{+}-Y^{+} \circ \bar{M}=O .
$$

As in [8], to obtain an asymptotic expansion of the solution of (12), we had better consider the following two cases separately:

(i) $\quad\left|x_{1}\right|<\exists$ constant $\left(\operatorname{Im} \xi_{n}\right)^{-1 / m q}$,

(ii) $\left|x_{1}\right|>\exists$ constant $\left(\operatorname{Im} \xi_{n}\right)^{-1 / m q}$.

We first consider the case (i). Let $a>>1$. In this section we define

$$
\begin{aligned}
& \omega(a)=\left\{\left(x, \xi^{\prime}\right) \in \mathbf{C}^{n} \times \mathbf{C}^{n-1} ; a^{3}\left(\operatorname{Im} \xi_{n}\right)^{-1 / m q}<\left|x_{1}\right|<a^{-1}, a\left|x^{\prime}\right|<1,\right. \\
&\left.a\left|\xi^{\prime \prime \prime}\right|<\operatorname{Im} \xi_{n}, a\left|\operatorname{Re} \xi_{n}\right|<\operatorname{Im} \xi_{n}\right\} .
\end{aligned}
$$

Let $f\left(x, \xi^{\prime}\right)=\sum_{k \in \mathbf{Z}_{+}}{ }^{*} f_{k}\left(x, \xi^{\prime}\right)$ be a formal series where $f_{k}\left(x, \xi^{\prime}\right) \in \mathcal{O}(\omega(a))$, and let $i, j \in \mathbf{R}$. We say that $f\left(x, \xi^{\prime}\right)$ belongs to $\mathcal{T}_{i, j}(\omega(a))$ if there exists some $b>0$ such that

$$
\left|f_{k}\left(x, \xi^{\prime}\right)\right| \leq b^{k+1} k !\left|x_{1}\right|^{i}\left(\operatorname{Im} \xi_{n}\right)^{j-k} \quad \text { on } \omega(a) .
$$

We define $\mathcal{T}_{i, j}(\omega(a))+\mathcal{T}_{k, \ell}(\omega(a))=\left\{\sum^{*}\left(f_{h}\left(x, \xi^{\prime}\right)+g_{h}\left(x, \xi^{\prime}\right)\right) ; \sum^{*} f_{h} \in\right.$ $\left.\mathcal{T}_{i, j}(\omega(a)) \sum^{*} g_{h} \in \mathcal{T}_{k, \ell}(\omega(a))\right\}, \mathcal{T}_{i, j}^{0}(\omega(a))=\left\{f\left(x, \xi^{\prime}\right)=\sum^{*} f_{k}\left(x, \xi^{\prime}\right) \in\right.$ $\left.\mathcal{T}_{i, j}(\omega(a)) ; f_{0}=0\right\}$, and $\mathcal{T}(\omega(a))=\bigcup_{i, j} \mathcal{T}_{i, j}(\omega(a))$. It is easy to see that $\mathcal{T}_{i-q, j-\frac{1}{m}}(\omega(a)) \subset \mathcal{T}_{i, j}(\omega(a))$. If $f \in \mathcal{T}_{i, j}(\omega(a)), g \in \mathcal{T}_{k, \ell}(\omega(a))$, then we have $f \circ g \in \mathcal{T}_{i+k, j+\ell}(\omega(a))$. Now we have the following

Lemma 3. Let a $>>$ 1. There exist $U^{ \pm}\left(x, \xi^{\prime}\right), \bar{L}^{\prime}\left(x, \xi^{\prime}\right) \quad \in$ $(\mathcal{T}(\omega(a)))^{m \times m}$ such that

(i) $\bar{L} \circ U^{+}=U^{+} \bar{L}^{\prime}, U^{ \pm} \circ U^{\mp}=I_{m}$, 


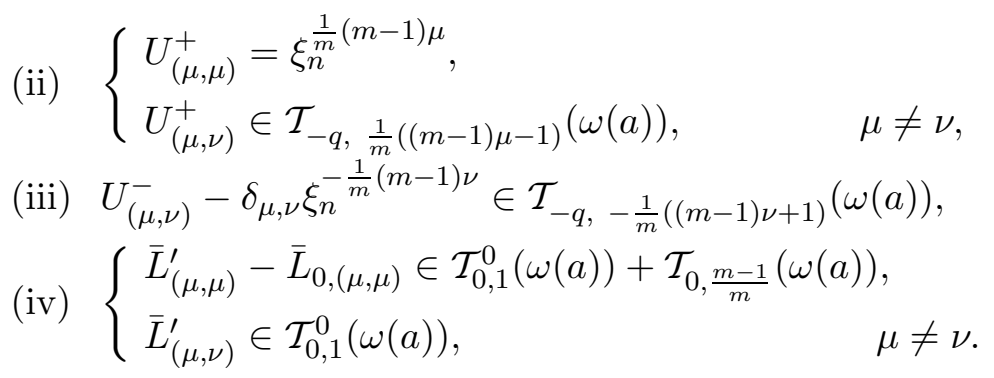

Proof. We first look for $U\left(x, \xi^{\prime}\right)=U^{+}\left(x, \xi^{\prime}\right) \in(\mathcal{O}(\omega(a)))^{m \times m}$. Let $\mu \neq \nu$. We define $U_{(\mu, \nu)}$ by

$$
\begin{gathered}
\left(\bar{L}_{0,(\mu, \mu)}-\bar{L}_{0,(\nu, \nu)}\right) U_{(\mu, \nu)}=U_{(\mu, \nu)} \sum_{\kappa \neq \nu} \xi_{n}^{-\frac{1}{m}(m-1) \nu} \bar{L}_{0,(\nu, \kappa)} U_{(\kappa, \nu)} \\
-\sum_{\kappa \neq \mu, \nu} \bar{L}_{0,(\mu, \kappa)} U_{(\kappa, \nu)}-\bar{L}_{0,(\mu, \nu)} \xi_{n}^{\frac{1}{m}(m-1) \nu} .
\end{gathered}
$$

We can solve (13) as follows: Let $U_{(\mu, \nu)}^{(i)}, i \in \mathbf{Z}_{+}, \quad \mu \neq \nu$ be successively determined by

$$
\begin{aligned}
& \left(\bar{L}_{0,(\mu, \mu)}-\bar{L}_{0,(\nu, \nu)}\right) U_{(\mu, \nu)}^{(i)} \\
= & U_{(\mu, \nu)}^{(i-1)} \sum_{\kappa \neq \nu} \xi_{n}^{-\frac{1}{m}(m-1) \nu} \bar{L}_{0,(\nu, \kappa)} U_{(\kappa, \nu)}^{(i-1)} \\
& -\sum_{\kappa \neq \mu, \nu} \bar{L}_{0,(\mu, \kappa)} U_{(\kappa, \nu)}^{(i-1)}-\bar{L}_{0,(\mu, \nu)} \xi_{n}^{\frac{1}{m}(m-1) \nu},
\end{aligned}
$$

where $U^{(-1)}=O$. If $\tilde{U}^{(i)}=U^{(i)}-U^{(i-1)}$ then we have

$$
\begin{aligned}
& \left(\bar{L}_{0,(\mu, \mu)}-\bar{L}_{0,(\nu, \nu)}\right) \tilde{U}_{(\mu, \nu)}^{(i)} \\
= & \sum_{\kappa \neq \nu} \xi_{n}^{-\frac{1}{m}(m-1) \nu} \bar{L}_{0,(\nu, \kappa)}\left(\tilde{U}_{(\mu, \nu)}^{(i-1)} U_{(\kappa, \nu)}^{(i-1)}+U_{(\mu, \nu)}^{(i-2)} \tilde{U}_{(\kappa, \nu)}^{(i-1)}\right)-\sum_{\kappa \neq \mu, \nu} \bar{L}_{0,(\mu, \kappa)} \tilde{U}_{(\kappa, \nu)}^{(i-1)}
\end{aligned}
$$

for $i \in \mathbf{N}$. By induction on $i$, we can easily prove

$$
\left|\tilde{U}_{(\mu, \nu)}^{(i)}\right| \leq\left(a^{2}\left|x_{1}\right|^{-q}\left(\operatorname{Im} \xi_{n}\right)^{-\frac{1}{m}}\right)^{i+1}\left(\operatorname{Im} \xi_{n}\right)^{\frac{1}{m}(m-1) \mu}
$$


replacing $a$ if necessary. We define

$$
U_{(\mu, \nu)}= \begin{cases}\xi_{n}^{\frac{1}{m}}(m-1) \mu & \mu=\nu \\ \sum_{i \in \mathbf{Z}_{+}} \tilde{U}_{(\mu, \nu)}^{(i)}(\text { convergent sum }), & \mu \neq \nu\end{cases}
$$

(These are not formal series but holomorphic functions). It follows that

$$
\left|U_{(\mu, \nu)}\right| \leq 2 a^{2}\left|x_{1}\right|^{-q}\left(\operatorname{Im} \xi_{n}\right)^{\frac{1}{m}((m-1) \mu-1)}, \quad \mu \neq \nu .
$$

Since $\left|\xi_{n}^{-\frac{1}{m}(m-1) \nu} U_{\mu, \nu}-\delta_{\mu, \nu}\right|<<1, U$ is a non-singular matrix. We can inductively define $U^{-}=\sum^{*} U_{i}^{-}$by

$$
U_{i}^{-}=\delta_{i, 0}\left(U^{+}\right)^{-1}-\sum_{\substack{i^{\prime}+\left|\alpha^{\prime}\right|=i \\ i^{\prime} \neq i}} \frac{1}{\alpha^{\prime} !} \partial_{\xi^{\prime}}^{\alpha^{\prime}} U_{i^{\prime}}^{-} \partial_{x^{\prime}}^{\alpha^{\prime}} U^{+}\left(U^{+}\right)^{-1}
$$

Here $\left(U^{+}\right)^{-1}$ denotes the usual inverse matrix of $U^{+}$, and $U^{-}$denotes the matrix (of formal series) satisfying $U^{ \pm} \circ U^{\mp}=I_{m}$. It is easy to see that

$$
U_{(\mu, \nu)}^{-}-\delta_{\mu, \nu} \xi_{n}^{-\frac{1}{m}(m-1) \nu} \in \mathcal{T}_{-q,-\frac{1}{m}((m-1) \nu+1)}(\omega(a))
$$

replacing $a$ if necessary.

We next define

$$
\begin{aligned}
\tilde{L}_{(\mu, \mu)}= & \bar{L}_{0,(\mu, \mu)}+\sum_{\kappa \neq \mu} \xi_{n}^{-\frac{1}{m}(m-1) \mu} \circ \bar{L}_{(\mu, \kappa)} \circ U_{(\kappa, \mu)} \\
\tilde{L}_{(\mu, \nu)}= & \xi_{n}^{-\frac{1}{m}(m-1) \mu} \circ\left\{\left(\bar{L}_{0,(\mu, \mu)} \circ U_{(\mu, \nu)}-U_{(\mu, \nu)} \circ \tilde{L}_{(\nu, \nu)}\right.\right. \\
& \left.+\sum_{\kappa \neq \mu, \nu} \bar{L}_{(\mu, \kappa)} \circ U_{(\kappa, \nu)}+\bar{L}_{(\mu, \nu)} \circ \xi_{n}^{\frac{1}{m}(m-1) \nu}\right\}, \quad \mu \neq \nu .
\end{aligned}
$$

From (14) it follows that

$$
\begin{aligned}
& \tilde{L}_{(\mu, \mu)} \in \bar{L}_{0,(\mu, \mu)}+\mathcal{T}_{-q, \frac{m-2}{m}}(\omega(a)), \\
& \tilde{L}_{(\mu, \nu)} \in \mathcal{T}_{-q, \frac{m-1}{m}}^{0}(\omega(a)), \quad \mu \neq \nu
\end{aligned}
$$


and

$$
\begin{array}{ll}
(\bar{L} \circ U)_{(\mu, \mu)} & \equiv(U \circ \tilde{L})_{(\mu, \mu)} \quad \text { modulo } \mathcal{T}_{0,1+\frac{1}{m}(m-1) \mu}^{0}(\omega(a)), \\
(\bar{L} \circ U)_{(\mu, \nu)} \equiv(U \circ \tilde{L})_{(\mu, \nu)} & \text { modulo } \mathcal{T}_{-q, \frac{1}{m}(m-1)(\mu+1)}^{0}(\omega(a)), \quad \mu \neq \nu,
\end{array}
$$

replacing $a$ if necessary.

We finally define $\bar{L}^{\prime}=U^{-} \circ \bar{L} \circ U^{+}$. It follows that $\bar{L}_{(\mu, \nu)}^{\prime} \equiv \tilde{L}_{(\mu, \nu)}$ modulo $\mathcal{T}_{0,1}^{0}(\omega(a))$, and we obtain the lemma.

Corollary. Let $a>>1$ and let $\bar{L}^{\prime \prime}=U^{-} \circ \partial_{x_{1}} U^{+}+\bar{L}^{\prime}$. Then we have

$$
\left\{\begin{array}{l}
\bar{L}_{(\mu, \mu)}^{\prime \prime}-\bar{L}_{0,(\mu, \mu)} \in \mathcal{T}_{0,1}^{0}(\omega(a))+\mathcal{T}_{0, \frac{m-1}{m}}(\omega(a)) \\
\bar{L}_{(\mu, \nu)}^{\prime \prime} \in \mathcal{T}_{0,1}^{0}(\omega(a))+\mathcal{T}_{-q-1,-\frac{1}{m}}(\omega(a)), \quad \mu \neq \nu
\end{array}\right.
$$

and $\partial_{x_{1}} U^{+}+\bar{L} \circ U^{+}-U^{+} \circ \bar{L}^{\prime \prime}=O$.

We next define some complex domains which will be necessary in the next section. Let $\theta^{\prime j}, \theta^{\prime \prime j} \in \mathbf{R},(1 \leq j \leq m)$ be such that

(i) $\quad \theta \in\left(\theta^{\prime m}, \theta^{\prime \prime m}\right) \subset\left(\theta^{\prime m-1}, \theta^{\prime \prime m-1}\right) \subset \cdots \subset\left(\theta^{\prime 1}, \theta^{\prime \prime 1}\right)$,

(ii) $\pi /\left(q_{j}+1\right)<\theta^{\prime \prime j}-\theta^{\prime j}<2 \pi /\left(2 q_{j}+1\right), 1 \leq j \leq m$,

(iii) there uniquely exists some $k_{j}$ belonging to $\mathbf{Z} \cap\left(\left(q_{j}+1\right) \theta^{\prime j} / \pi,\left(q_{j}+\right.\right.$ 1) $\left.\theta^{\prime \prime j} / \pi\right)$ for $1 \leq j \leq m$.

Note that for any $\theta$ we can always choose such numbers. In fact it is trivial if $m=1$. Assume that $m_{0} \geq 2$ and that we can choose the above numbers if $m=m_{0}-1$. Let $m=m_{0}$. By assumption we can choose $\theta^{\prime j}, \theta^{\prime \prime j}$ suitably for $1 \leq j \leq m-1$. If $q_{m}=q_{m-1}$, then we may take $\theta^{\prime m}=\theta^{\prime m-1}, \theta^{\prime \prime m}=\theta^{\prime \prime m-1}$. If $q_{m}>q_{m-1}$, then $\pi /\left(q_{m}+1\right)<\theta^{\prime \prime m-1}-\theta^{\prime m-1}$, and we can choose a subset $\left(\theta^{\prime m}, \theta^{\prime \prime m}\right)$ of $\left(\theta^{\prime m-1}, \theta^{\prime \prime m-1}\right)$ such that (i) - (iii) are true.

It is easy to see that in each interval $\left[\theta^{\prime j}, \theta^{\prime \prime j}\right]$ the function $f(\tau)=$ $\pm \sin \left(\left(q_{j}+1\right) \tau\right)$ attains its maximum (resp. minimum) at a uniquely determined point $\tau=\theta^{+, j}$ (resp. $\left.\theta^{-, j}\right)$.

Let $1<<a<<1 / r<<C_{1}<<\cdots<<C_{m}$. We define

$$
\tilde{\Omega}^{\prime j}\left(r, C_{j}\right)=\left\{\left(x, \xi^{\prime}\right) \in \mathbf{C}^{n} \times \mathbf{C}^{n-1} ; \operatorname{Im} \xi_{n}>2^{-1} C_{j}^{5 m q},\right.
$$




$$
\begin{aligned}
& (-1)^{k_{j}} \operatorname{Re}\left(x_{1}^{q_{j}+1}\right)+r^{-1}\left|\operatorname{Im}\left(x_{1}^{q_{j}+1}\right)\right| \\
& >2^{-1} C_{j}^{4}\left(\operatorname{Im} \xi_{n}\right)^{-\left(q_{j}+1\right) / m q}, \\
& \left|\operatorname{Re}\left(x_{1}^{q_{j}+1}\right)\right|+r^{-1}\left|\operatorname{Im}\left(x_{1}^{q_{j}+1}\right)\right|<C_{j}^{-q_{j}-1}, \arg x_{1} \in\left(\theta^{\prime j}, \theta^{\prime \prime j}\right), \\
& \left.C_{j}^{\frac{1}{10}}\left|x^{\prime}\right|<1, C_{j}^{\frac{1}{10}}\left|\xi^{\prime \prime \prime}\right|<\operatorname{Im} \xi_{n}, C_{j}^{\frac{1}{10}}\left|\operatorname{Re} \xi_{n}\right|<\operatorname{Im} \xi_{n}\right\}, \\
\Omega^{\prime j}\left(r, C_{j}\right)=\{ & \left(x, \xi^{\prime}\right) \in \mathbf{C}^{n} \times \mathbf{C}^{n-1} ; \operatorname{Im} \xi_{n}>C_{j}^{5 m q}, \\
& (-1)^{k_{j}} \operatorname{Re}\left(x_{1}^{q_{j}+1}\right)+r^{-1}\left|\operatorname{Im}\left(x_{1}^{q_{j}+1}\right)\right|>C_{j}^{4}\left(\operatorname{Im} \xi_{n}\right)^{-\left(q_{j}+1\right) / m q}, \\
& \left|\operatorname{Re}\left(x_{1}^{q_{j}+1}\right)\right|+r^{-1}\left|\operatorname{Im}\left(x_{1}^{q_{j}+1}\right)\right|<C_{j}^{-q_{j}-1}, \arg x_{1} \in\left(\theta^{\prime j}, \theta^{\prime \prime j}\right), \\
& \left.C_{j}\left|x^{\prime}\right|<1, C_{j}\left|\xi^{\prime \prime \prime}\right|<\operatorname{Im} \xi_{n}, C_{j}\left|\operatorname{Re} \xi_{n}\right|<\operatorname{Im} \xi_{n}\right\}
\end{aligned}
$$

for $1 \leq j \leq m$. It is easy to see that $\Omega^{\prime j}\left(r, C_{j}\right) \subset \tilde{\Omega}^{\prime j}\left(r, C_{j}\right)$, and $\Omega^{\prime j}\left(r, C_{j}^{\prime}\right) \subset$ $\Omega^{\prime j}\left(r, C_{j}\right)$ if $C_{j}^{\prime} \geq C_{j}$. Furthermore we may assume

$$
\Omega^{\prime m}\left(r, C_{m}\right) \subset \Omega^{\prime m-1}\left(r, C_{m-1}\right) \subset \cdots \subset \Omega^{\prime 1}\left(r, C_{1}\right)
$$

(See the figure below).

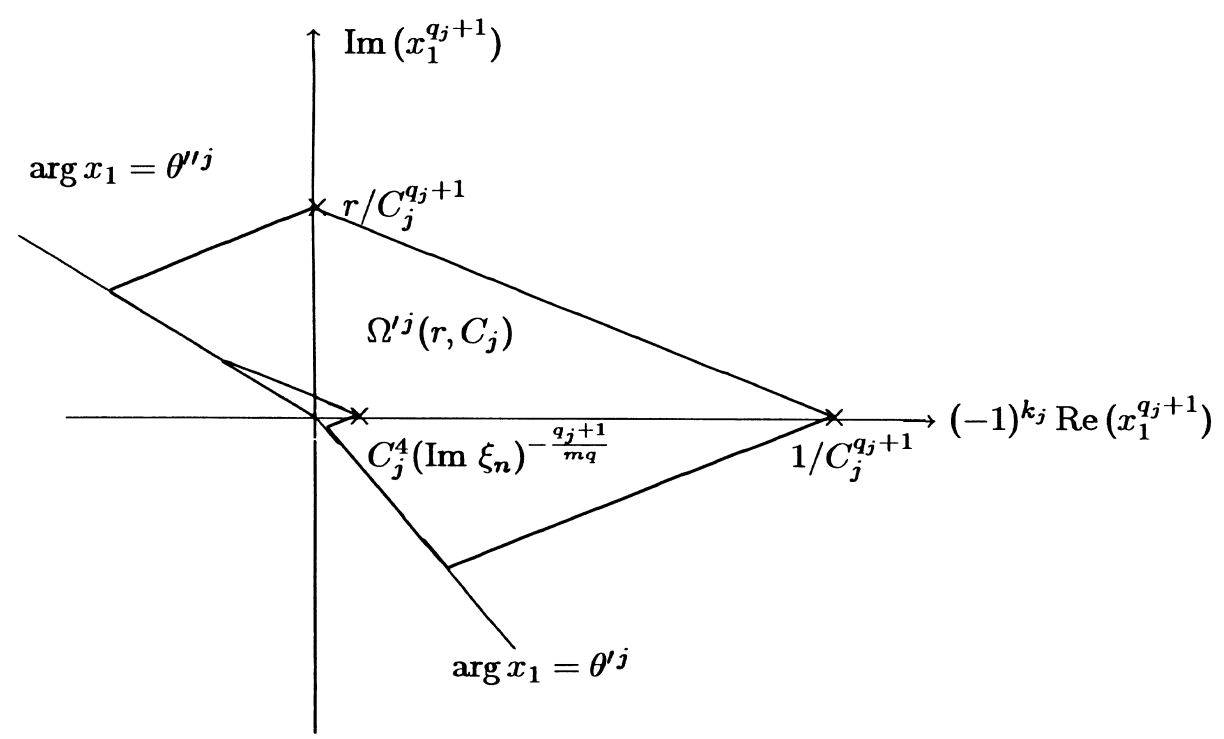


We define $\phi_{j, k}\left(x_{1}, t, \xi^{\prime}\right)=-\int_{t}^{x_{1}}\left(\bar{L}_{0,(j, j)}^{\prime \prime}\left(s, x^{\prime}, \xi^{\prime}\right)-\bar{L}_{0,(k, k)}^{\prime \prime}\left(s, x^{\prime}, \xi^{\prime}\right)\right) d s$ for $1 \leq j, k \leq m$. Let $x_{1}^{ \pm, j}$ be the point defined by

$$
\left\{\begin{array}{l}
\left|\operatorname{Re}\left(\left(x_{1}^{ \pm, j}\right)^{q_{j}+1}\right)\right|+r^{-1}\left|\operatorname{Im}\left(\left(x_{1}^{ \pm, j}\right)^{q_{j}+1}\right)\right|=C_{j}^{-q_{j}-1} \\
\arg x_{1}^{ \pm, j}=\theta^{ \pm, j}
\end{array}\right.
$$

LEMMA 4. Let $x_{1}$ be a complex number such that $\left(x, \xi^{\prime}\right) \in \tilde{\Omega}^{\prime j}\left(r, C_{j}\right)$ for some $\left(x^{\prime}, \xi^{\prime}\right)$. We can connect $x_{1}^{ \pm, j}$ and $x_{1}$ by a continuous curve $\delta^{ \pm, j}\left(x_{1}\right)$ with length at most $C_{j}^{-9 / 10}$ such that

(i) if $\left(x, \xi^{\prime}\right) \in \tilde{\Omega}^{\prime j}\left(r, C_{j}\right)\left(\right.$ resp. $\left.\Omega^{\prime j}\left(r, C_{j}\right)\right)$ and $t \in \delta^{ \pm, j}\left(x_{1}\right) \backslash\left\{x_{1}^{ \pm, j}\right\}$, then we have $\left(t, x^{\prime}, \xi^{\prime}\right) \in \tilde{\Omega}^{\prime j}\left(r, C_{j}\right)\left(\right.$ resp. $\left.\Omega^{\prime j}\left(r, C_{j}\right)\right)$,

(ii) if $\left(x, \xi^{\prime}\right) \in \tilde{\Omega}^{\prime j}\left(r, C_{j}\right), t \in \delta^{\mp, j}\left(x_{1}\right)$, $\pm \operatorname{Im} a_{j}\left(x^{*}\right)<0$, and $j \leq k$, then $\operatorname{Re} \phi_{j, k}\left(x, t, \xi^{\prime}\right) \leq 0$,

(iii) if $\left(x, \xi^{\prime}\right) \in \tilde{\Omega}^{\prime k}\left(r, C_{k}\right), t \in \delta^{ \pm, k}\left(x_{1}\right), \pm \operatorname{Im} a_{k}\left(x^{*}\right)<0$, and $k \leq j$, then $\operatorname{Re} \phi_{j, k}\left(x, t, \xi^{\prime}\right) \leq 0$.

Proof. If $\pm \operatorname{Im} x_{1}^{q_{j}} \geq 0$, we let $\gamma^{ \pm, j}\left(x_{1}\right)$ be the line segment between $\left(x_{1}^{ \pm, j}\right)^{q_{j}+1}$ and $x_{1}^{q_{j}+1}$. If $\pm \operatorname{Im} x_{1}^{q_{j}} \leq 0$, we define $d \in \mathbf{R}$ by $\operatorname{Re}\left(x_{1}^{q_{j}+1}\right)+$ $(-1)^{k_{j}} r^{-1}\left|\operatorname{Im}\left(x_{1}^{q_{j}+1}\right)\right|=d$, and let $\gamma^{ \pm, j}\left(x_{1}\right)$ be the union of the two line segments, one from $\left(x_{1}^{ \pm, j}\right)^{q_{j}+1}$ to $d$, and the other from $d$ to $x_{1}^{q_{j}+1}$. In both cases we define $\delta^{ \pm, j}\left(x_{1}\right)=\left\{t \in \mathbf{C} ; t^{q_{j}+1} \in \gamma^{ \pm, j}\left(x_{1}\right)\right\}$ (See the figure below).

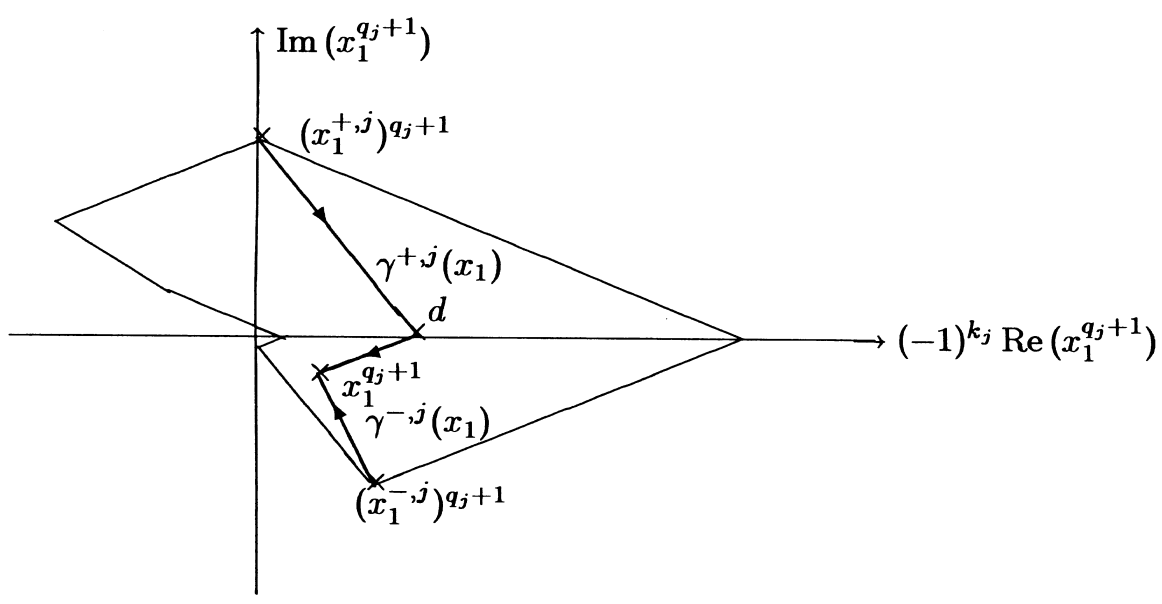


Then statement (i) is trivial, and we prove (ii). We have $\operatorname{Re} \phi_{j, k}\left(x, t, \xi^{\prime}\right)$ $=\mathrm{I}+\mathrm{II}+\mathrm{III}+\mathrm{IV}$, where

$$
\begin{aligned}
\mathrm{I}= & \operatorname{Im} \frac{x_{1}^{q_{j}+1}-t^{q_{j}+1}}{q_{j}+1} \cdot \operatorname{Im} a_{j}\left(0, x^{\prime}, \xi^{\prime}\right)-\operatorname{Im} \frac{x_{1}^{q_{k}+1}-t^{q_{k}+1}}{q_{k}+1} \cdot \operatorname{Im} a_{k}\left(0, x^{\prime}, \xi^{\prime}\right), \\
\mathrm{II}= & -\operatorname{Re} \frac{x_{1}^{q_{j}+1}-t^{q_{j}+1}}{q_{j}+1} \cdot \operatorname{Re} a_{j}\left(0, x^{\prime}, \xi^{\prime}\right) \\
& +\operatorname{Re} \frac{x_{1}^{q_{k}+1}-t^{q_{k}+1}}{q_{k}+1} \cdot \operatorname{Re} a_{k}\left(0, x^{\prime}, \xi^{\prime}\right), \\
\mathrm{III}= & -\operatorname{Re}\left(\int_{t}^{x_{1}}\left(s_{1}^{q_{j}} a_{j}{ }^{\prime}\left(s, x^{\prime}, \xi^{\prime}\right)-s_{1}^{q_{k}} a_{k}{ }^{\prime}\left(s, x^{\prime}, \xi^{\prime}\right)\right) d s\right), \\
\mathrm{IV}= & -\operatorname{Re}\left(\int_{t}^{x_{1}}\left(\bar{L}_{0,(j, j)}^{\prime \prime \prime}\left(s, x^{\prime}, \xi^{\prime}\right)-\bar{L}_{0,(k, k)}^{\prime \prime \prime}\left(s, x^{\prime}, \xi^{\prime}\right)\right) d s\right) .
\end{aligned}
$$

Here $a_{j}{ }^{\prime}\left(x, \xi^{\prime}\right)=a_{j}\left(x, \xi^{\prime}\right)-a_{j}\left(0, x^{\prime}, \xi^{\prime}\right)$, and $\bar{L}_{0,(j, j)}^{\prime \prime \prime}\left(x, \xi^{\prime}\right)=\bar{L}_{0,(j, j)}^{\prime \prime}\left(x, \xi^{\prime}\right)-$ $x_{1}^{q_{j}} a_{j}\left(x, \xi^{\prime}\right)$. If $j=k$, the statement is trivial, so we consider the case $j<k$. We want to show that

$$
I \leq-a^{-3}\left|x_{1}-t\right|\left(\left|x_{1}\right|+|t|\right)^{q_{j}} \operatorname{Im} \xi_{n}
$$

We remind the reader that $q_{j} \leq q_{k}$. If $q_{j}=q_{k}$, then (16) is trivial, and we consider the case $q_{j}<q_{k}$. Let $0 \leq \ell \leq q_{j}$. Since $\arg x_{1}, \arg t \in\left(\theta^{\prime j}, \theta^{\prime \prime j}\right)$, we have

$$
\arg \left(x_{1}^{q_{j}-\ell} t^{\ell} e^{\frac{q_{j}\left(\theta^{\prime \prime j}-\theta^{\prime j}\right)}{2 \sqrt{-1}}}\right) \in\left(-q_{j} \pi /\left(2 q_{j}+1\right), q_{j} \pi /\left(2 q_{j}+1\right)\right),
$$

and thus

$$
\begin{aligned}
\left|\sum_{0 \leq \ell \leq q_{j}} x_{1}^{q_{j}-\ell} t^{\ell}\right| & \geq \sum_{0 \leq \ell \leq q_{j}} \operatorname{Re}\left(x_{1}^{q_{j}-\ell} t^{\ell} e^{\frac{q_{j}\left(\theta^{\prime \prime j}-\theta^{\prime j}\right)}{2 \sqrt{-1}}}\right) \\
& \geq \cos \left(\frac{q_{j} \pi}{2 q_{j}+1}\right) \sum_{0 \leq \ell \leq q_{j}}\left|x_{1}^{q_{j}-\ell} t^{\ell}\right| .
\end{aligned}
$$

It follows that

$$
\pm \operatorname{Im}\left(x_{1}^{q_{j}+1}-t^{q_{j}+1}\right) \leq-\left|x_{1}^{q_{j}+1}-t^{q_{j}+1}\right| / 2 a \leq-a^{-2}\left|x_{1}-t\right|\left(\left|x_{1}\right|+|t|\right)^{q_{j}},
$$


and

$$
\left|\operatorname{Im}\left(x_{1}^{q_{k}+1}-t^{q_{k}+1}\right)\right| \leq\left|x_{1}^{q_{k}+1}-t^{q_{k}+1}\right| \leq\left|x_{1}-t\right|\left(\left|x_{1}\right|+|t|\right)^{q_{j}+1} .
$$

It follows that

$$
\begin{aligned}
\mathrm{I} \leq & \left|x_{1}-t\right|\left(\left|x_{1}\right|+|t|\right)^{q_{j}} \\
& \cdot\left(-a^{-2} \operatorname{Im} a_{j}\left(0, x^{\prime}, \xi^{\prime}\right)+a\left(\left|x_{1}\right|+|t|\right) \operatorname{Im} a_{k}\left(0, x^{\prime}, \xi^{\prime}\right)\right) \\
& \leq-a^{-3}\left|x_{1}-t\right|\left(\left|x_{1}\right|+|t|\right)^{q_{j}} \operatorname{Im} \xi_{n} .
\end{aligned}
$$

On the other hand, it is easy to see that

$$
\text { II, III, IV } \leq a^{-4}\left|x_{1}-t\right|\left(\left|x_{1}\right|+|t|\right)^{q_{j}} \operatorname{Im} \xi_{n},
$$

and we obtain (ii). The proof of (iii) is similar.

Corollary. Assume one of the following conditions:

(i) $\left(x, \xi^{\prime}\right) \in \Omega^{\prime j}\left(r, C_{j}\right), t \in \delta^{\mp, j}\left(x_{1}\right), \pm \operatorname{Im} a_{j}\left(x^{*}\right)<0$, and $j \leq k$.

(ii) $\left(x, \xi^{\prime}\right) \in \Omega^{\prime k}\left(r, C_{k}\right), t \in \delta^{ \pm, k}\left(x_{1}\right)$, $\pm \operatorname{Im} a_{k}\left(x^{*}\right)<0$, and $j \geq k$.

Then we have

$$
\left|\partial_{x^{\prime}}^{\alpha^{\prime}} \partial_{\xi^{\prime}}^{\beta^{\prime}} \exp \left(\phi_{j, k}\left(x, t, \xi^{\prime}\right)\right)\right| \leq C_{j}^{\frac{1}{5}\left|\alpha^{\prime}+\beta^{\prime}\right|} \alpha^{\prime} ! \beta^{\prime} !\left(\operatorname{Im} \xi_{n}\right)^{-\left|\beta^{\prime}\right|} .
$$

\section{Study of Ordinary Differential Equations}

We can find a solution $Y^{+}$of (12) in several steps. We formally define $C_{0}=C_{1}$ and $\Omega^{\prime 0}\left(r, C_{0}\right)=\Omega^{\prime 1}\left(r, C_{1}\right)$. The essential part is the following

Proposition 3. Let $0 \leq \lambda \leq m$. Then there exist

$$
V_{i}^{\lambda}, W_{i}^{\lambda}, \bar{M}_{i}^{\lambda} \in\left(\mathcal{O}\left(\Omega^{\prime \lambda}\left(r, C_{\lambda}\right)\right)\right)^{m \times m}, \quad i \in \mathbf{Z}_{+}
$$

such that on $\Omega^{\prime \lambda}\left(r, C_{\lambda}\right)$ we have

(i) $\left|\partial_{x^{\prime}}^{\alpha^{\prime}} \partial_{\xi^{\prime}}^{\beta^{\prime}}\left(V_{i,(\mu, \nu)}^{\lambda}-\delta_{i, 0} \delta_{\mu, \nu}\right)\right|,\left|\partial_{x^{\prime}}^{\alpha^{\prime}} \partial_{\xi^{\prime}}^{\beta^{\prime}}\left(W_{i,(\mu, \nu)}^{\lambda}-\delta_{i, 0} \delta_{\mu, \nu}\right)\right|$ 


$$
\begin{aligned}
& \leq 300 m^{2} r^{2}(3 m)^{\lambda} \sum_{k \leq i}\left(i-k+\left|\alpha^{\prime}+\beta^{\prime}\right|\right) ! \\
& \times C_{\lambda}^{2 i-2 k+\frac{1}{4}\left|\alpha^{\prime}+\beta^{\prime}\right|} r^{k(m+1-\lambda)}\left(\operatorname{Im} \xi_{n}\right)^{-i+k-\left|\beta^{\prime}\right|},
\end{aligned}
$$

(ii) $\left|\partial_{x^{\prime}}^{\alpha^{\prime}} \partial_{\xi^{\prime}}^{\beta^{\prime}}\left(\bar{M}_{i,(\mu, \nu)}^{\lambda}-\bar{L}_{i,(\mu, \nu)}^{\prime \prime}\right)\right|$

$$
\begin{aligned}
& \leq \sum_{k \leq i}\left(i-k+\left|\alpha^{\prime}+\beta^{\prime}\right|\right) ! C_{\lambda}^{2 i-2 k+\frac{1}{4}\left|\alpha^{\prime}+\beta^{\prime}\right|+1} r^{k(m+1-\lambda)}\left(\operatorname{Im} \xi_{n}\right)^{-i+k-\left|\beta^{\prime}\right|} \\
& \times\left(1-\delta_{i, 0}+\left|x_{1}\right|^{-q-1}\left(\operatorname{Im} \xi_{n}\right)^{-\frac{1}{m}}\right),
\end{aligned}
$$

(iii) $\bar{M}_{i,(\mu, \nu)}^{\lambda}=\delta_{i, 0} \delta_{\mu, \nu} \bar{L}_{0,(\mu, \nu)}^{\prime \prime}, \quad \mu \leq \lambda$ or $\nu \leq \lambda$,

(iv) $V^{\lambda} \circ W^{\lambda}=W^{\lambda} \circ V^{\lambda}=I_{m}$,

(v) $\partial_{x_{1}} V^{\lambda}+\bar{L}^{\prime \prime} \circ V^{\lambda}-V^{\lambda} \circ \bar{M}^{\lambda}=O$.

Here $V^{\lambda}$ denotes the formal series $V^{\lambda}=\sum_{i}^{*} V_{i}^{\lambda}$, and $V^{\lambda}$ transforms $\bar{L}^{\prime \prime}$ into $\bar{M}^{\lambda}$, where

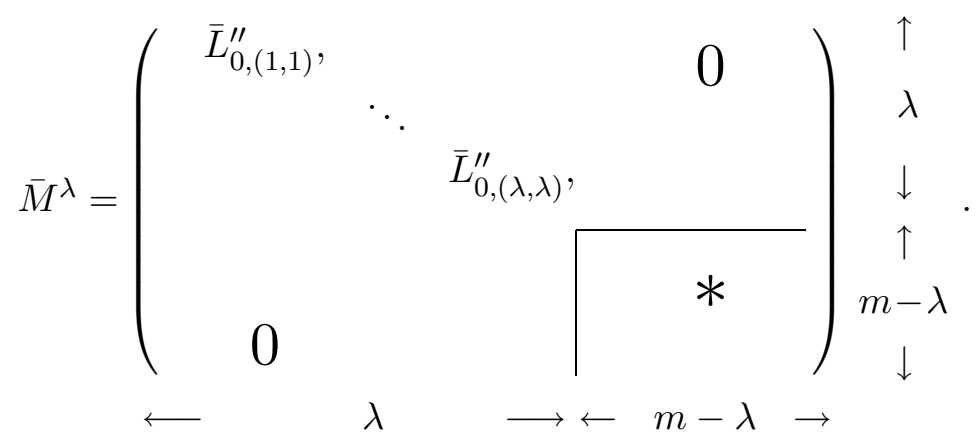

In other words, $\bar{V}^{\lambda}$ diagonalizes $\bar{L}^{\prime \prime}$ up to the $\lambda$-th column and the $\lambda$-th row. Note that $\bar{M}^{m}$ is a diagonal matrix. Therefore we can diagonalize $\bar{L}^{\prime \prime}$ if Proposition 3 is true for $1 \leq \lambda \leq m$. We can prove Proposition 3 by induction on $\lambda$. If $\lambda=0$, then we may choose $V^{\lambda}=W^{\lambda}=I_{m}, \bar{M}^{\lambda}=$ $\bar{L}^{\prime \prime}$, and the statements are trivial. Assume that $1 \leq \lambda_{0} \leq m$ and that the statements are true if $0 \leq \lambda \leq \lambda_{0}-1$. Let $\pm \operatorname{Im} a_{\lambda_{0}}\left(x^{*}\right)<0$. Then we can prove the case $\lambda=\lambda_{0}$ in two steps. Roughly speaking, we first diagonalize the $\lambda$-th column, and then the $\lambda$-th row. The first step is the following 
LEMma 5. There exist

$$
V_{i}^{\prime \lambda}, W_{i}^{\prime \lambda}, \bar{M}_{i}^{\prime \lambda} \in\left(\mathcal{O}\left(\Omega^{\prime \lambda}\left(r, C_{\lambda}\right)\right)\right)^{m \times m}, i \in \mathbf{Z}_{+}
$$

such that on $\Omega^{\prime \lambda}\left(r, C_{\lambda}\right)$ we have

(i) $\left|\partial_{x^{\prime}}^{\alpha^{\prime}} \partial_{\xi^{\prime}}^{\beta^{\prime}}\left(V_{i,(\mu, \nu)}^{\prime \lambda}-\delta_{i, 0} \delta_{\mu, \nu}\right)\right|,\left|\partial_{x^{\prime}}^{\alpha^{\prime}} \partial_{\xi^{\prime}}^{\beta^{\prime}}\left(W_{i,(\mu, \nu)}^{\prime \lambda}-\delta_{i, 0} \delta_{\mu, \nu}\right)\right|$

$\leq 100 m^{2} r^{2} \sum_{k \leq i}\left(i-k+\left|\alpha^{\prime}+\beta^{\prime}\right|\right) ! C_{\lambda}^{2 i-2 k+\frac{1}{4}\left|\alpha^{\prime}+\beta^{\prime}\right|}$

$\times\left(4 r^{m+2-\lambda}\right)^{k}\left(\operatorname{Im} \xi_{n}\right)^{-i+k-\left|\beta^{\prime}\right|}$,

(ii) $\left|\partial_{x^{\prime}}^{\alpha^{\prime}} \partial_{\xi^{\prime}}^{\beta^{\prime}}\left(\bar{M}_{i,(\mu, \nu)}^{\prime \lambda}-\bar{L}_{i,(\mu, \nu)}^{\prime \prime}\right)\right|$

$\leq \sum_{k \leq i}\left(i-k+\left|\alpha^{\prime}+\beta^{\prime}\right|\right) ! C_{\lambda}^{2 i-2 k+\frac{1}{4}\left|\alpha^{\prime}+\beta^{\prime}\right|+1}\left(4 r^{m+2-\lambda}\right)^{k}\left(\operatorname{Im} \xi_{n}\right)^{-i+k-\left|\beta^{\prime}\right|}$

$\times\left(1-\delta_{i, 0}+\left|x_{1}\right|^{-q-1}\left(\operatorname{Im} \xi_{n}\right)^{-\frac{1}{m}}\right)$,

(iii) $V_{i,(\mu, \nu)}^{\prime \lambda}, W_{i,(\mu, \nu)}^{\prime \lambda}=\delta_{i, 0} \delta_{\mu, \nu}, \quad \mu \leq \lambda-1$ or $\nu \neq \lambda$,

(iv) $\bar{M}_{i,(\mu, \nu)}^{\prime \lambda}=\delta_{i, 0} \delta_{\mu, \nu} \bar{L}_{0,(\mu, \nu)}^{\prime \prime}, \quad \mu \leq \lambda-1$ or $\nu \leq \lambda$,

(v) $V^{\prime \lambda} \circ W^{\prime \lambda}=W^{\prime \lambda} \circ V^{\prime \lambda}=I_{m}$,

(vi) $\partial_{x_{1}} V^{\prime \lambda}+\bar{M}^{\lambda-1} \circ V^{\prime \lambda}-V^{\prime \lambda} \circ \bar{M}^{\prime \lambda}=O$.

This means that we have

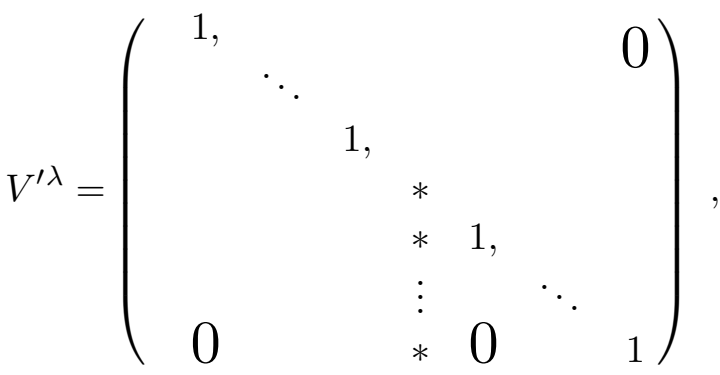

$$
\begin{aligned}
& \frown \\
& \lambda
\end{aligned}
$$


and

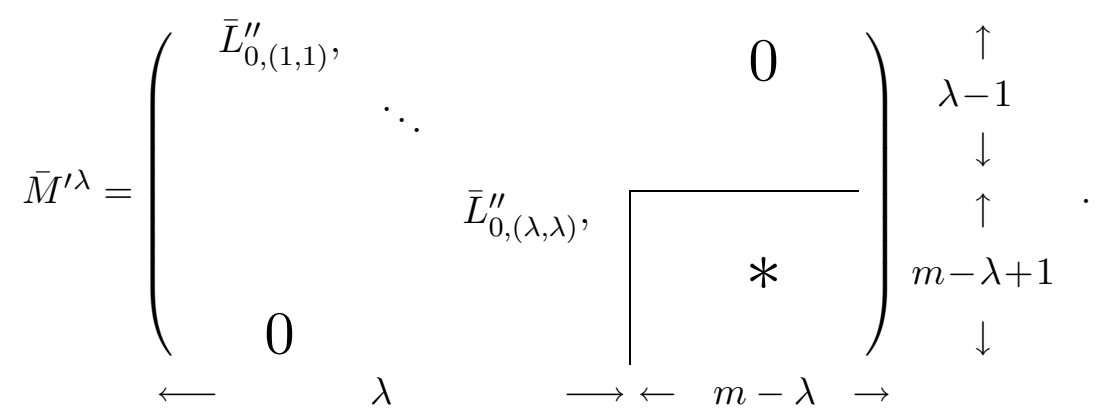

In other words, $\bar{V}^{\prime \lambda}$ diagonalizes the $\lambda$-th column of $\bar{M}^{\lambda-1}$. Calculating the $(\mu, \lambda)$-component of (vi) for $\lambda \leq \mu \leq m$, it follows that

$$
\partial_{x_{1}} V_{(\mu, \lambda)}^{\prime \lambda}+\sum_{1 \leq \kappa \leq m} \bar{M}_{(\mu, \kappa)}^{\lambda-1} \circ V_{(\kappa, \lambda)}^{\prime \lambda}-V_{(\mu, \lambda)}^{\prime \lambda} \circ \bar{L}_{0,(\lambda, \lambda)}^{\prime \prime}=0 .
$$

If $\mu \geq \lambda$ or $\nu \geq \lambda+1$, calculating the $(\mu, \nu)$-component of (vi) we obtain

$$
\bar{M}_{(\mu, \nu)}^{\lambda-1}-\sum_{1 \leq \kappa \leq m} V_{(\mu, \kappa)}^{\prime \lambda} \circ \bar{M}_{(\kappa, \nu)}^{\prime \lambda}=0 .
$$

Note that the other components of the left hand side of (vi) always vanish. We shall see that if $V^{\prime \lambda}$ satisfies (17) and it is invertible, we can define $\bar{M}^{\prime \lambda}$ by (18). We can rewrite (17) in the form

$$
\begin{gathered}
\partial_{x_{1}} V_{i,(\mu, \lambda)}^{\prime \lambda}+\sum_{\substack{\lambda \leq \kappa \leq m \\
i^{\prime}+i^{\prime \prime}+\left|\alpha^{\prime}\right|=i}} \frac{1}{\alpha^{\prime} !} \partial_{\xi^{\prime}}^{\alpha^{\prime}} \bar{M}_{i^{\prime},(\mu, \kappa)}^{\lambda-1} \partial_{x^{\prime}}^{\alpha^{\prime}} V_{i^{\prime \prime},(\kappa, \lambda)}^{\prime \lambda} \\
-\sum_{i^{\prime \prime}+\left|\alpha^{\prime}\right|=i} \frac{1}{\alpha^{\prime} !} \partial_{\xi^{\prime}}^{\alpha^{\prime}} V_{i^{\prime \prime},(\mu, \lambda)}^{\prime \lambda} \partial_{x^{\prime}}^{\alpha^{\prime}} \bar{L}_{0,(\lambda, \lambda)}^{\prime \prime}=0, i \in \mathbf{Z}_{+} .
\end{gathered}
$$

Let us solve (17) by successive approximation:

Sublemma 1. Let $i, j \in \mathbf{Z}_{+}, \lambda \leq \mu \leq m$. We denote by $\rho^{ \pm, \lambda}\left(x_{1}\right)$ the length of $\delta^{ \pm, \lambda}\left(x_{1}\right)$. There exists $\tilde{V}_{i}^{\prime \lambda, j} \in\left(\mathcal{O}\left(\Omega^{\prime \lambda}\left(r, C_{\lambda}\right)\right)\right)^{m \times m}, i \in \mathbf{Z}_{+}$, such that

$$
\begin{aligned}
& \left|\partial_{x^{\prime}}^{\alpha^{\prime}} \partial_{\xi^{\prime}}^{\beta^{\prime}}\left(\tilde{V}_{i,(\mu, \lambda)}^{\prime \lambda, j}-\delta_{i, 0} \delta_{j, 0} \delta_{\mu, \lambda}\right)\right| \leq r^{2} 2^{-j} \sum_{k+\ell \leq i} \frac{\left(i-k+\left|\alpha^{\prime}+\beta^{\prime}\right|\right) !}{\ell !} \\
\times & C_{\lambda}^{2 i-2 k-\frac{3}{2} \ell+\frac{1}{4}\left|\alpha^{\prime}+\beta^{\prime}\right|}\left(2 r^{m+2-\lambda}\right)^{k}\left(\rho^{ \pm, \lambda}\left(x_{1}\right)\right)^{\ell}\left(\operatorname{Im} \xi_{n}\right)^{-i+k+\ell-\left|\beta^{\prime}\right|}
\end{aligned}
$$


on $\Omega^{\prime \lambda}\left(r, C_{\lambda}\right)$, and $V^{\prime \lambda, j}=\sum_{0 \leq j^{\prime} \leq j} \tilde{V}^{\prime \lambda, j^{\prime}}$ satisfies

$$
\partial_{x_{1}} V_{i,(\mu, \lambda)}^{\prime \lambda, j}+\left(\bar{L}_{0,(\mu, \mu)}^{\prime \prime}-\bar{L}_{0,(\lambda, \lambda)}^{\prime \prime}\right) V_{i^{\prime \prime},(\mu, \lambda)}^{\prime \lambda}=F_{i,(\mu, \lambda)}^{\prime \lambda, j}=\sum_{1 \leq k \leq 4} F_{i,(\mu, \lambda)}^{\prime \lambda, j, k}
$$

where

$$
\begin{aligned}
& F_{i,(\mu, \lambda)}^{\prime \lambda, j, 1}=-\sum_{\lambda \leq \kappa \leq m}\left(\bar{M}_{0,(\mu, \kappa)}^{\lambda-1}-\delta_{\mu, \kappa} \bar{L}_{0,(\mu, \mu)}^{\prime \prime}\right) V_{i^{\prime \prime},(\kappa, \lambda)}^{\prime \lambda, j-1}, \\
& F_{i,(\mu, \lambda)}^{\lambda, j, 2}=-\sum_{\substack{\lambda \leq \kappa \leq m \\
i,+\left|\alpha^{\prime}\right|=i \\
i^{\prime \prime} \neq i}} \frac{1}{\alpha^{\prime} !} \partial_{\xi^{\prime}}^{\alpha^{\prime}} \bar{M}_{0,(\mu, \kappa)}^{\lambda-1} \partial_{x^{\prime}}^{\alpha^{\prime}} V_{i^{\prime \prime},(\kappa, \lambda)}^{\prime \lambda}, \\
& F_{i,(\mu, \lambda)}^{\lambda, j, 3}=-\sum_{\substack{\lambda \leq \kappa \leq m \\
i^{\prime}+i^{\prime \prime}+\left|\alpha^{\prime}\right|=i \\
i^{\prime} \neq 0}} \frac{1}{\alpha^{\prime} !} \partial_{\xi^{\prime}}^{\alpha^{\prime}} \bar{M}_{i^{\prime},(\mu, \kappa)}^{\lambda-1} \partial_{x^{\prime}}^{\alpha^{\prime}} V_{i^{\prime \prime},(\kappa, \lambda)}^{\prime \lambda}, \\
& F_{i,(\mu, \lambda)}^{\prime \lambda, j, 4}=\sum_{\substack{i^{\prime \prime}+\left|\alpha^{\prime}\right|=i \\
i^{\prime \prime} \neq i}} \frac{1}{\alpha^{\prime} !} \partial_{\xi^{\prime}}^{\alpha^{\prime}} V_{i^{\prime \prime},(\mu, \lambda)}^{\prime \lambda} \partial_{x^{\prime}}^{\alpha^{\prime}} \bar{L}_{0,(\lambda, \lambda)}^{\prime \prime}
\end{aligned}
$$

on $\Omega^{\prime \lambda}\left(r, C_{\lambda}\right)$. Here we define $V^{\prime \lambda,-1}=0$.

Proof. If $i=j=0$, then we may take $\tilde{V}_{i}^{\prime \lambda, j}=I_{m}$. Assume that $\left(i_{0}, j_{0}\right) \in \mathbf{Z}_{+}{ }^{2} \backslash\{(0,0)\}$ and that if $(i, j) \in \mathbf{Z}_{+}{ }^{2}$ satisfies either $i \leq i_{0}-1$ or $i=i_{0}, j \leq j_{0}-1$, then the statements are true. Let us consider the case $(i, j)=\left(i_{0}, j_{0}\right)$. We can prove

$$
\begin{aligned}
& \left|\partial_{x^{\prime}}^{\alpha^{\prime}} \partial_{\xi^{\prime}}^{\beta^{\prime}}\left(F_{i,(\mu, \lambda)}^{\prime \lambda, j, h}-F_{i,(\mu, \lambda)}^{\prime \lambda, j-1, h}\right)\right| \leq r^{2} 2^{-j} \sum_{k+\ell \leq i} \frac{\left(i-k+\left|\alpha^{\prime}+\beta^{\prime}\right|\right) !}{\ell !} \\
\times & C_{\lambda}^{2 i-2 k-\frac{3}{2} \ell+\frac{1}{4}\left|\alpha^{\prime}+\beta^{\prime}\right|}\left(2 r^{m+2-\lambda}\right)^{k}\left(\rho^{ \pm, \lambda}\left(x_{1}\right)\right)^{\ell}\left(\operatorname{Im} \xi_{n}\right)^{-i+k+\ell-\left|\beta^{\prime}\right|} \\
\times & \left\{C_{\lambda}^{\frac{1}{5}}\left(1-\delta_{i, 0}+\left|x_{1}\right|^{-q-1}\left(\operatorname{Im} \xi_{n}\right)^{-\frac{1}{m}}\right)+4 m n C_{\lambda}^{-\frac{31}{20}}\left(1-\delta_{k+\ell, i}\right) \operatorname{Im} \xi_{n}\right\}
\end{aligned}
$$

for $1 \leq h \leq 4$. Let us prove (20) for the case $h=2,3$ (The proof of the other cases are easier). Since $C_{\lambda-1}<<C_{\lambda}$, from (15) and the assumption of induction (on $\lambda$ ) we may assume

$$
\left|\partial_{x^{\prime}}^{\alpha^{\prime}} \partial_{\xi^{\prime}}^{\beta^{\prime}} \bar{M}_{0,(\mu, \kappa)}^{\lambda-1}\right| \leq \alpha^{\prime} ! \beta^{\prime} ! C_{\lambda}^{\frac{1}{20}\left(\left|\alpha^{\prime}+\beta^{\prime}\right|+1\right)}\left(\operatorname{Im} \xi_{n}\right)^{1-\left|\beta^{\prime}\right|}
$$


on $\Omega^{\prime \lambda-1}\left(r, C_{\lambda-1}\right)\left(\supset \Omega^{\prime \lambda}\left(r, C_{\lambda}\right)\right)$. Let $\left(x, \xi^{\prime}\right) \in \Omega^{\prime \lambda}\left(r, C_{\lambda}\right)$. Summing up (19) for $j \in \mathbf{Z}_{+}$(for each $i^{\prime \prime} \leq i-1$ ), we obtain

$$
\begin{gathered}
\left|\partial_{x^{\prime}}^{\alpha^{\prime}} \partial_{\xi^{\prime}}^{\beta^{\prime}}\left(V_{i^{\prime \prime},(\mu, \lambda)}^{\prime \lambda}-\delta_{i^{\prime \prime}, 0} \delta_{\mu, \lambda}\right)\right| \leq 2 r^{2} \sum_{k+\ell \leq i-1} \frac{\left(i^{\prime \prime}-k+\left|\alpha^{\prime}+\beta^{\prime}\right|\right) !}{\ell !} \\
\times C_{\lambda}^{2 i^{\prime \prime}-2 k-\frac{3}{2} \ell+\frac{1}{4}\left|\alpha^{\prime}+\beta^{\prime}\right|}\left(2 r^{m+2-\lambda}\right)^{k}\left(\rho^{ \pm, \lambda}\left(x_{1}\right)\right)^{\ell}\left(\operatorname{Im} \xi_{n}\right)^{-i^{\prime \prime}+k+\ell-\left|\beta^{\prime}\right|}
\end{gathered}
$$

if $\left|\alpha^{\prime}\right| \neq 0$. From (21) and (22), we have

$$
\begin{aligned}
&\left|\partial_{x^{\prime}}^{\alpha^{\prime}} \partial_{\xi^{\prime}}^{\beta^{\prime}} F_{i,(\mu, \lambda)}^{\prime \lambda, j, 2}\right| \\
& \leq \sum_{(23)} \frac{\alpha^{\prime} ! \beta^{\prime} !}{\alpha^{\prime(1)} ! \alpha^{\prime(2)} ! \beta^{\prime(1)} ! \beta^{\prime(2)} ! \gamma^{\prime} !}\left|\partial_{x^{\prime}}^{\alpha^{\prime(1)}} \partial_{\xi^{\prime}}^{\beta^{\prime(1)}+\gamma^{\prime}} \bar{M}_{0,(\mu, \kappa)}^{\lambda-1} \partial_{x^{\prime}}^{\alpha^{\prime(2)}+\gamma^{\prime}} \partial_{\xi^{\prime}}^{\beta^{\prime(2)}} V_{i^{\prime \prime},(\kappa, \lambda)}^{\prime \lambda}\right| \\
& \leq 2 r^{2} \sum_{(23)} \sum_{k+\ell \leq i^{\prime \prime}} \frac{\alpha^{\prime} ! \beta^{\prime} !}{\alpha^{\prime(1)} ! \alpha^{\prime(2) !} \beta^{\prime(1)} ! \beta^{\prime(2)} ! \gamma^{\prime} !} \alpha^{\prime(1) !} ! \beta^{\prime(1)} ! \gamma^{\prime} !\left(2 C_{\lambda}^{\frac{1}{20}}\right)^{\left|\alpha^{\prime(1)}+\beta^{\prime(1)}+\gamma^{\prime}\right|+1} \\
& \times\left(\operatorname{Im} \xi_{n}\right)^{1-\left|\beta^{\prime(1)}+\gamma^{\prime}\right|} \frac{\left(i^{\prime \prime}-k+\left|\alpha^{\prime(2)}+\beta^{\prime(2)}+\gamma^{\prime}\right|\right) !}{\ell !} \\
& \times C_{\lambda}^{2 i^{\prime \prime}-2 k-\frac{3}{2} \ell+\frac{1}{4}\left|\alpha^{\prime(2)}+\beta^{\prime(2)}+\gamma^{\prime}\right|} \\
& \times\left(2 r^{m+2-\lambda}\right)^{k}\left(\rho^{ \pm, \lambda}\left(x_{1}\right)\right)^{\ell}\left(\operatorname{Im} \xi_{n}\right)^{-i^{\prime \prime}+k+\ell-\left|\beta^{\prime(2)}\right|}
\end{aligned}
$$

where the first summation is taken for

$$
i^{\prime \prime}+\left|\gamma^{\prime}\right|=i, i^{\prime \prime} \neq i, \alpha^{\prime(1)}+\alpha^{\prime(2)}=\alpha^{\prime}, \beta^{\prime(1)}+\beta^{\prime(2)}=\beta^{\prime}, \lambda \leq \kappa \leq m .
$$

It follows that

$$
\begin{aligned}
& \left|\partial_{x^{\prime}}^{\alpha^{\prime}} \partial_{\xi^{\prime}}^{\beta^{\prime}} F_{i,(\mu, \lambda)}^{\prime \lambda, j, 2}\right| \\
& \leq \sum_{(23)} \sum_{k+\ell \leq i-1} 2 r^{2} C_{\lambda}^{-\frac{1}{10}\left|\alpha^{\prime(1)}+\beta^{\prime(1)}\right|-\frac{33}{20}\left|\gamma^{\prime}\right|+\frac{1}{10}} \frac{\left(i-k+\left|\alpha^{\prime}+\beta^{\prime}\right|\right)}{\ell !} \\
& \times C_{\lambda}^{2 i-2 k-\frac{3}{2} \ell+\frac{1}{4}\left|\alpha^{\prime}+\beta^{\prime}\right|}\left(2 r^{m+2-\lambda}\right)^{k}\left(\rho^{ \pm, \lambda}\left(x_{1}\right)\right)^{\ell}\left(\operatorname{Im} \xi_{n}\right)^{1-i+k+\ell-\left|\beta^{\prime}\right|} \\
& \leq \sum_{k+\ell \leq i} 4 m n r^{2} C_{\lambda}^{-\frac{31}{20}}\left(1-\delta_{k+\ell, i}\right) \operatorname{Im} \xi_{n} \frac{\left(i-k+\left|\alpha^{\prime}+\beta^{\prime}\right|\right) !}{\ell !} \\
& \times C_{\lambda}^{2 i-2 k-\frac{3}{2} \ell+\frac{1}{4}\left|\alpha^{\prime}+\beta^{\prime}\right|}\left(2 r^{m+2-\lambda}\right)^{k}\left(\rho^{ \pm, \lambda}\left(x_{1}\right)\right)^{\ell}\left(\operatorname{Im} \xi_{n}\right)^{-i+k+\ell-\left|\beta^{\prime}\right|}
\end{aligned}
$$


Since $F_{i,(\mu, \lambda)}^{\prime \lambda, j, h}-F_{i,(\mu, \lambda)}^{\prime \lambda, j-1, h}=\delta_{j, 0} F_{i,(\mu, \lambda)}^{\prime \lambda, j, h}$ for $2 \leq h \leq 4$, we obtain (20) for $h=2$.

On the other hand, from (15) and the assumption of induction (on $\lambda$ ) we have

$$
\begin{aligned}
& \quad\left|\partial_{x^{\prime}}^{\alpha^{\prime}} \partial_{\xi^{\prime}}^{\beta^{\prime}} \bar{M}_{i^{\prime},(\mu, \kappa)}^{\lambda-1}\right| \leq \sum_{k^{\prime} \leq i^{\prime}}\left(i^{\prime}-k^{\prime}\right) ! \alpha^{\prime} ! \beta^{\prime} ! C_{\lambda}^{\frac{1}{20}\left(i^{\prime}-k^{\prime}+\left|\alpha^{\prime}+\beta^{\prime}\right|+1\right)} r^{k^{\prime}(m+2-\lambda)} \\
& \times\left(\operatorname{Im} \xi_{n}\right)^{-i^{\prime}+k-\left|\beta^{\prime}\right|}\left\{1+\left|x_{1}\right|^{-q-1}\left(\operatorname{Im} \xi_{n}\right)^{-\frac{1}{m}}+\delta_{k^{\prime}, 0}\left(\operatorname{Im} \xi_{n}\right)^{\frac{m-1}{m}}\right\}
\end{aligned}
$$

if $i^{\prime} \geq 1$. It follows that

$$
\begin{aligned}
& \left|\partial_{x^{\prime}}^{\alpha^{\prime}} \partial_{\xi^{\prime}}^{\beta^{\prime}} F_{i,(\mu, \lambda)}^{\prime \lambda, j, 3}\right|
\end{aligned}
$$

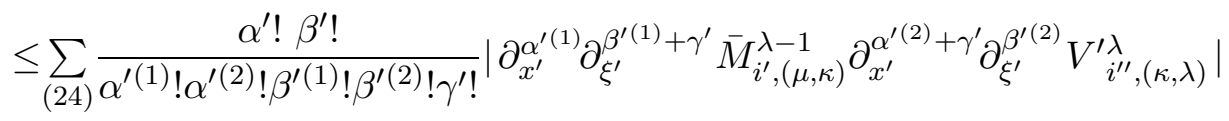

$$
\begin{aligned}
& \leq \sum_{(24)(25)} \sum_{\alpha^{\prime(1)} ! \alpha^{\prime(2)} ! \beta^{\prime(1)} ! \beta^{\prime(2)} ! \gamma^{\prime} !}^{\alpha^{\prime} ! \beta^{\prime} !}\left(i^{\prime}-k^{\prime}\right) ! \alpha^{\prime(1)} ! \beta^{\prime(1)} ! \gamma^{\prime} ! \\
& \times\left(2 C_{\lambda}^{\frac{1}{20}}\right)^{i^{\prime}-k^{\prime}+\left|\alpha^{\prime(1)}+\beta^{\prime(1)}+\gamma^{\prime}\right|+1} \\
& \times r^{k^{\prime}(m+2-\lambda)}\left(\operatorname{Im} \xi_{n}\right)^{-i^{\prime}+k-\left|\beta^{\prime(1)}+\gamma^{\prime}\right|} \\
& \times\left\{1+\left|x_{1}\right|^{-q-1}\left(\operatorname{Im} \xi_{n}\right)^{-\frac{1}{m}}+\delta_{k^{\prime}, 0}\left(\operatorname{Im} \xi_{n}\right)^{\frac{m-1}{m}}\right\} \\
& \times \frac{\left(i^{\prime \prime}-k^{\prime \prime}+\left|\alpha^{\prime(2)}+\beta^{\prime(2)}+\gamma^{\prime}\right|\right) !}{\ell^{\prime \prime} !} 2 C_{\lambda}^{2 i^{\prime \prime}-2 k^{\prime \prime}-\frac{3}{2} \ell^{\prime \prime}+\frac{1}{4}\left|\alpha^{(2)}+\beta^{(2)}+\gamma^{\prime}\right|} \\
& \times\left(2 r^{m+2-\lambda}\right)^{k^{\prime \prime}}\left(\rho^{ \pm, \lambda}\left(x_{1}\right)\right)^{\ell^{\prime \prime}}\left(\operatorname{Im} \xi_{n}\right)^{-i^{\prime \prime}+k^{\prime \prime}+\ell^{\prime \prime}-\left|\beta^{\prime(2)}\right|} \\
& \leq \sum_{(24)(25)} \sum_{\lambda} 2 C_{\lambda}^{-\frac{19}{10}\left(i^{\prime}-k^{\prime}\right)-\frac{1}{10}\left(\left|\alpha^{\prime(1)}+\beta^{\prime(1)}+\gamma^{\prime}\right|-1\right)} 2^{-k^{\prime}} \frac{\left(i-k^{\prime}-k^{\prime \prime}+\left|\alpha^{\prime}+\beta^{\prime}\right|\right) !}{\ell^{\prime \prime} !} \\
& \times C_{\lambda}^{2 i-2\left(k^{\prime}+k^{\prime \prime}\right)-\frac{3}{2} \ell^{\prime \prime}+\frac{1}{4}\left|\alpha^{\prime}+\beta^{\prime}\right|}\left(2 r^{m+2-\lambda}\right)^{k^{\prime}+k^{\prime \prime}}\left(\rho^{ \pm, \lambda}\left(x_{1}\right)\right)^{\ell^{\prime \prime}} \\
& \times\left(\operatorname{Im} \xi_{n}\right)^{-i+k^{\prime}+k^{\prime \prime}+\ell^{\prime \prime}-\left|\beta^{\prime}\right|} \\
& \times\left\{1+\left|x_{1}\right|^{-q-1}\left(\operatorname{Im} \xi_{n}\right)^{-\frac{1}{m}}+\delta_{k^{\prime}, 0}\left(\operatorname{Im} \xi_{n}\right)^{\frac{m-1}{m}}\right\} .
\end{aligned}
$$

Here the summations are taken for

(24) $i^{\prime}+i^{\prime \prime}+\left|\gamma^{\prime}\right|=i, i^{\prime} \neq 0, \alpha^{\prime(1)}+\alpha^{\prime(2)}=\alpha^{\prime}, \beta^{\prime(1)}+\beta^{\prime(2)}=\beta^{\prime}, \lambda \leq \kappa \leq m$ 
and

$$
k^{\prime} \leq i^{\prime}, k^{\prime \prime}+\ell^{\prime \prime} \leq i^{\prime \prime}
$$

respectively. We let $k^{\prime}+k^{\prime \prime}=k, \ell^{\prime \prime}=\ell$. Note that if $\delta_{k^{\prime}, 0}=1$, then we have $i^{\prime}-k^{\prime}=i^{\prime} \geq 1, k+\ell \neq i$ and $1-\delta_{k+\ell, i}=1$. It follows that $\delta_{k^{\prime}, 0} \leq 1-\delta_{k+\ell, i}$ and we obtain

$$
\begin{aligned}
& \left|\partial_{x^{\prime}}^{\alpha^{\prime}} \partial_{\xi^{\prime}}^{\beta^{\prime}} F_{i,(\mu, \lambda)}^{\prime \lambda, j, 3}\right| \leq 8 m C_{\lambda}^{\frac{1}{10}} \sum_{k+\ell \leq i} \frac{\left(i-k+\left|\alpha^{\prime}+\beta^{\prime}\right|\right) !}{\ell !} \\
\times & C_{\lambda}^{2 i-2 k-\frac{3}{2} \ell+\frac{1}{4}\left|\alpha^{\prime}+\beta^{\prime}\right|}\left(2 r^{m+2-\lambda}\right)^{k}\left(\rho^{ \pm, \lambda}\left(x_{1}\right)\right)^{\ell}\left(\operatorname{Im} \xi_{n}\right)^{-i+k+\ell-\left|\beta^{\prime}\right|} \\
\times & \left\{1+\left|x_{1}\right|^{-q-1}\left(\operatorname{Im} \xi_{n}\right)^{-\frac{1}{m}}+C_{\lambda}^{-\frac{9}{5}}\left(1-\delta_{k+\ell, i}\right) \operatorname{Im} \xi_{n}\right\} .
\end{aligned}
$$

In this way we can prove $(20)$ for $1 \leq h \leq 4$.

We have assumed that $\pm \operatorname{Im} a_{\lambda_{0}}\left(x^{*}\right)<0$, and now we define

$$
\tilde{V}_{i,(\mu, \lambda)}^{\lambda, j}\left(x, \xi^{\prime}\right)=\delta_{i, 0} \delta_{j, 0} \delta_{\mu, \lambda}+\int_{\delta^{ \pm, \lambda}\left(x_{1}\right)} \exp \left(\phi_{\mu, \lambda}\left(x, t, \xi^{\prime}\right)\right) \tilde{F}_{\mu, \lambda}^{\prime \lambda, j}\left(t, x^{\prime}, \xi^{\prime}\right) d t
$$

where $\tilde{F}_{\mu, \lambda}^{\prime \lambda, j}=F_{\mu, \lambda}^{\prime \lambda, j}-F_{\mu, \lambda}^{\prime \lambda, j-1}$. From (20) and Corollary of Lemma 4, it follows that

$$
\begin{aligned}
& \left|\partial_{x^{\prime}}^{\alpha^{\prime}} \partial_{\xi^{\prime}}^{\beta^{\prime}}\left(\tilde{V}_{i,(\mu, \lambda)}^{\lambda, j}\left(x, \xi^{\prime}\right)-\delta_{i, 0} \delta_{j, 0} \delta_{\mu, \lambda}\right)\right| \leq \sum_{\substack{\alpha^{\prime(1)}+\alpha^{\prime(2)}=\alpha^{\prime} \\
\beta^{\prime(1)}+\beta^{\prime(2)}=\beta^{\prime}}} \frac{\alpha^{\prime} ! \beta^{\prime \prime} !}{\alpha^{\prime(1)} ! \alpha^{\prime(2)} ! \beta^{\prime(1) ! \beta^{\prime(2)} !}} \\
& \times \int_{\delta^{ \pm, \lambda}\left(x_{1}\right)}\left|\partial_{x^{\prime}}^{\alpha^{\prime(1)}} \partial_{\xi^{\prime}}^{\beta^{\prime(1)}} \exp \left(\phi_{\mu, \lambda}\left(x, t, \xi^{\prime}\right)\right)\left\|\partial_{x^{\prime}}^{\alpha^{\prime(2)}} \partial_{\xi^{\prime}}^{\beta^{\prime(2)}} \tilde{F}_{\lambda, \nu}^{\lambda, j}\left(t, x^{\prime}, \xi^{\prime}\right)\right\| d t\right| \\
& \leq r^{2} 2^{-j} \sum_{(26)} \frac{\alpha^{\prime} ! \beta^{\prime} !}{\alpha^{\prime(1)} ! \alpha^{(2)} ! \beta^{\prime(1)} ! \beta^{\prime(2) !}} \\
& \times \int_{\delta^{ \pm, \lambda}\left(x_{1}\right)} C_{\lambda}^{\frac{1}{5}\left|\alpha^{\prime(1)}+\beta^{\prime(1)}\right|} \alpha^{\prime(1)} ! \beta^{\prime(1)} !\left(\operatorname{Im} \xi_{n}\right)^{-\left|\beta^{\prime(1)}\right|} \\
& \times \frac{\left(i-k+\left|\alpha^{\prime(2)}+\beta^{\prime(2)}\right|\right) !}{\ell !} C_{\lambda}^{2 i-2 k-\frac{3}{2} \ell+\frac{1}{4}\left|\alpha^{(2)}+\beta^{\prime(2)}\right|}\left(2 r^{m+2-\lambda}\right)^{k}\left(\rho^{ \pm, \lambda}(t)\right)^{\ell} \\
& \times\left(\operatorname{Im} \xi_{n}\right)^{-i+k+\ell-\mid \beta^{\prime(2)}} \mid\left\{C_{\lambda}^{\frac{1}{5}}\left(1+|t|^{-q-1}\left(\operatorname{Im} \xi_{n}\right)^{-\frac{1}{m}}\right)\right. \\
& \left.+4 m n C_{\lambda}^{-\frac{31}{20}}\left(1-\delta_{k+\ell, i}\right) \operatorname{Im} \xi_{n}\right\}|d t|
\end{aligned}
$$




$$
\begin{aligned}
& \leq r^{2} 2^{-j+2} \sum_{(26)} C_{\lambda}^{-\frac{1}{20}\left|\alpha^{\prime(1)}+\beta^{\prime(1)}\right|} \frac{\left(i-k+\left|\alpha^{\prime}+\beta^{\prime}\right|\right) !}{\ell !} C_{\lambda}^{2 i-2 k-\frac{3}{2} \ell+\frac{1}{4}\left|\alpha^{\prime}+\beta^{\prime}\right|} \\
& \times\left(2 r^{m+2-\lambda}\right)^{k}\left(\operatorname{Im} \xi_{n}\right)^{-i+k+\ell-\left|\beta^{\prime}\right|} \int_{\delta^{ \pm, \lambda}\left(x_{1}\right)}\left(\rho^{ \pm, \lambda}(t)\right)^{\ell} \\
& \times\left\{C_{\lambda}^{\frac{1}{5}}\left(1+|t|^{-q-1}\left(\operatorname{Im} \xi_{n}\right)^{-\frac{1}{m}}\right)+4 m n C_{\lambda}^{-\frac{31}{20}}\left(1-\delta_{k+\ell, i}\right) \operatorname{Im} \xi_{n}\right\}|d t|
\end{aligned}
$$

where the summation is taken for

$$
\alpha^{\prime(1)}+\alpha^{\prime(2)}=\alpha^{\prime}, \beta^{\prime(1)}+\beta^{\prime(2)}=\beta^{\prime}, k+\ell \leq i .
$$

It is easy to see that $\int_{\delta^{ \pm, \lambda}\left(x_{1}\right)}\left(\rho^{ \pm, \lambda}(t)\right)^{\ell}|d t|=(\ell+1)^{-1}\left(\rho^{ \pm, \lambda}\left(x_{1}\right)\right)^{\ell+1}$, and

$$
\begin{aligned}
& \int_{\delta^{ \pm, \lambda}\left(x_{1}\right)}\left(\rho^{ \pm, \lambda}(t)\right)^{\ell}|t|^{-q-1}\left(\operatorname{Im} \xi_{n}\right)^{-\frac{1}{m}}|d t| \\
\leq & \left(\rho^{ \pm, \lambda}\left(x_{1}\right)\right)^{\ell}\left(\operatorname{Im} \xi_{n}\right)^{-\frac{1}{m}} \int_{\delta^{ \pm, \lambda}\left(x_{1}\right)}|t|^{-q-1}|d t| \\
\leq & \left(\rho^{ \pm, \lambda}\left(x_{1}\right)\right)^{\ell} C_{\lambda}^{\frac{1}{10}}\left|x_{1}\right|^{-q}\left(\operatorname{Im} \xi_{n}\right)^{-\frac{1}{m}} \leq C_{\lambda}^{-1}\left(\rho^{ \pm, \lambda}\left(x_{1}\right)\right)^{\ell} .
\end{aligned}
$$

Since $\rho^{ \pm, \lambda}\left(x_{1}\right) \leq C_{\lambda}^{-\frac{9}{10}}$, we also have

$$
\begin{aligned}
& \int_{\delta^{ \pm, \lambda}\left(x_{1}\right)}\left(\rho^{ \pm, \lambda}(t)\right)^{\ell} \\
\times & \left\{C_{\lambda}^{\frac{1}{5}}\left(1+|t|^{-q-1}\left(\operatorname{Im} \xi_{n}\right)^{-\frac{1}{m}}\right)+4 m n C_{\lambda}^{-\frac{31}{20}}\left(1-\delta_{k+\ell, i}\right) \operatorname{Im} \xi_{n}\right\}|d t| \\
\leq & 2 C_{\lambda}^{-\frac{7}{10}}\left(\rho^{ \pm, \lambda}\left(x_{1}\right)\right)^{\ell}+(\ell+1)^{-1} 4 m n C_{\lambda}^{-\frac{31}{20}}\left(1-\delta_{k+\ell, i}\right)\left(\rho^{ \pm, \lambda}\left(x_{1}\right)\right)^{\ell+1} .
\end{aligned}
$$

We obtain (19) immediately from this.

We define $V^{\prime \lambda, j}=\sum_{0 \leq j^{\prime} \leq j} \tilde{V}^{\prime \lambda, j^{\prime}}$, and $V^{\prime \lambda}=\lim _{j \rightarrow \infty} V^{\prime \lambda, j}$. Then we have the following

Corollary. Let $i, j \in \mathbf{Z}_{+}$. We have

$$
\begin{gathered}
\quad\left|\partial_{x^{\prime}}^{\alpha^{\prime}} \partial_{\xi^{\prime}}^{\beta^{\prime}}\left(V_{i,(\mu, \nu)}^{\prime \lambda}-\delta_{i, 0} \delta_{\mu, \nu}\right)\right| \leq 4 r^{2} \sum_{k \leq i}\left(i-k+\left|\alpha^{\prime}+\beta^{\prime}\right|\right) ! \\
\times\left(2 C_{\lambda}^{2}\right)^{i-k}\left(2 C_{\lambda}^{\frac{1}{4}}\right)^{\left|\alpha^{\prime}+\beta^{\prime}\right|}\left(2 r^{m+2-\lambda}\right)^{k}\left(\operatorname{Im} \xi_{n}\right)^{-i+k-\left|\beta^{\prime}\right|}
\end{gathered}
$$

on $\Omega^{\prime \lambda}\left(r, C_{\lambda}\right)$. 
Proof. If $\mu<\lambda$ or $\nu \neq \lambda$, the statement is trivial. Let $\lambda \leq \mu \leq m$, $\nu=\lambda$. Since $\rho^{ \pm, \lambda}\left(x_{1}\right) \leq C_{\lambda}^{-\frac{9}{10}}$ we obtain

$$
\begin{aligned}
& \left|\partial_{x^{\prime}}^{\alpha^{\prime}} \partial_{\xi^{\prime}}^{\beta^{\prime}}\left(\tilde{V}_{i,(\mu, \lambda)}^{\prime \lambda}-\delta_{i, 0} \delta_{\mu, \lambda}\right)\right| \leq 2 r^{2} \sum_{k+\ell \leq i}\left(i-k-\ell+\left|\alpha^{\prime}+\beta^{\prime}\right|\right) ! \\
\times & 2^{i-k+\left|\alpha^{\prime}+\beta^{\prime}\right|} C_{\lambda}^{2 i-2 k-\frac{3}{2} \ell+\frac{1}{4}\left|\alpha^{\prime}+\beta^{\prime}\right|}\left(2 r^{m+2-\lambda}\right)^{k} C_{\lambda}^{-\frac{9}{10} \ell}\left(\operatorname{Im} \xi_{n}\right)^{-i+k+\ell-\left|\beta^{\prime}\right|} \\
\leq & 4 r^{2} \sum_{k \leq i}\left(i-k+\left|\alpha^{\prime}+\beta^{\prime}\right|\right) !\left(2 C_{\lambda}^{2}\right)^{i-k} \\
\times & \left(2 C_{\lambda}^{\frac{1}{4}}\right)^{\left|\alpha^{\prime}+\beta^{\prime}\right|}\left(2 r^{m+2-\lambda}\right)^{k}\left(\operatorname{Im} \xi_{n}\right)^{-i+k-\left|\beta^{\prime}\right|} .
\end{aligned}
$$

Increasing $C_{\lambda}$ (but not replacing $r>0$ ) if necessary, we obtain the following

Sublemma 2. There exist

$$
W_{i}^{\prime \lambda}, \bar{M}_{i}^{\prime \lambda} \in\left(\mathcal{O}\left(\Omega^{\prime \lambda}\left(r, C_{\lambda}\right)\right)\right)^{m \times m}, i \in \mathbf{Z}_{+}
$$

such that on $\Omega^{\prime \lambda}\left(r, C_{\lambda}\right)$ we have (18) and

(i) $\left|\partial_{\xi^{\prime}}^{\alpha^{\prime}}\left(W_{i,(\mu, \nu)}^{\prime \lambda}-\delta_{i, 0} \delta_{\mu, \nu}\right)\right|$ $\leq 100 m^{2} r^{2} \sum_{k \leq i}\left(i-k+\left|\alpha^{\prime}\right|\right) ! C_{\lambda}^{2 i-2 k+\frac{1}{4}\left|\alpha^{\prime}\right|}\left(4 r^{m+2-\lambda}\right)^{k}\left(\operatorname{Im} \xi_{n}\right)^{-i+k-\left|\alpha^{\prime}\right|}$,

(ii) $\left|\bar{M}_{i,(\mu, \nu)}^{\prime \lambda}-\bar{L}_{i,(\mu, \nu)}^{\prime \prime}\right| \leq \sum_{k \leq i}(i-k) ! C_{\lambda}^{2 i-2 k+1}\left(4 r^{m+2-\lambda}\right)^{k}\left(\operatorname{Im} \xi_{n}\right)^{-i+k}$ $\times\left(1-\delta_{i .0}+\left|x_{1}\right|^{-q-1}\left(\operatorname{Im} \xi_{n}\right)^{-\frac{1}{m}}\right)$,

(iii) $V^{\prime \lambda} \circ W^{\prime \lambda}=W^{\prime \lambda} \circ V^{\prime \lambda}=I_{m}$.

Proof. Since $\left|V_{0,(\mu, \nu)}^{\prime \lambda}-\delta_{\mu, \nu}\right| \leq 4 r^{2},\left(V_{0}^{\prime \lambda}\right)^{-1}=\sum_{j \in \mathbf{Z}_{+}}\left(I_{m}-V_{0}^{\prime \lambda}\right)^{j}$ is well-defined . Here $\left(V_{0}^{\prime \lambda}\right)^{-1}$ denotes the usual inverse matrix of $V_{0}^{\prime \lambda}$, and we denote it by $W_{0}^{\prime \lambda}$. We have $\left|W_{0,(\mu, \nu)}^{\prime \lambda}-\delta_{\mu, \nu}\right| \leq 8 r^{2}$. Increasing $C_{\lambda}$ if necessary, we have

$$
\left|\partial_{\xi^{\prime}}^{\alpha^{\prime}}\left(W_{0,(\mu, \nu)}^{\prime \lambda}-\delta_{\mu, \nu}\right)\right| \leq 8 r^{2} \alpha^{\prime} ! C_{\lambda}^{\frac{1}{10}\left(\left|\alpha^{\prime}\right|+1\right)}\left(\operatorname{Im} \xi_{n}\right)^{-\left|\alpha^{\prime}\right|}
$$


(We do not change other constants such as $r, \theta_{\lambda}^{\prime}, \theta_{\lambda}^{\prime \prime}$ ). If $i \geq 1$, then we define $W_{i}^{\prime \lambda}$ inductively by

$$
W_{i}^{\prime \lambda}=-\sum_{\substack{i^{\prime}+i^{\prime \prime}+\left|\alpha^{\prime}\right|=i \\ i^{\prime \prime} \neq i}} \frac{1}{\alpha^{\prime} !} \partial_{\xi^{\prime}}^{\alpha^{\prime}} W_{i^{\prime \prime}}^{\prime \lambda} \partial_{x^{\prime}}^{\alpha^{\prime}} V_{i^{\prime}}^{\prime \lambda} W_{0}^{\prime \lambda}
$$

Let $\mu \geq \lambda, \nu \geq \lambda+1$. From (18) it follows that $\bar{M}_{(\mu, \nu)}^{\prime \lambda}=\left(W^{\prime \lambda} \circ\right.$ $\left.\bar{M}^{\lambda-1}\right)_{(\mu, \nu)}$. Sublemma 2 follows immediately from these relations.

Therefore we have proved Lemma 5 , replacing $C_{\lambda}>>1$ if necessary.

The second step of the proof of Proposition 3 is the following

Lemma 6. Let $C_{\lambda}>>1$. There exist

$$
V_{i}^{\prime \prime \lambda}, W_{i}^{\prime \prime \lambda}, \bar{M}_{i}^{\prime \prime \lambda} \in\left(\mathcal{O}\left(\Omega^{\prime \lambda}\left(r, C_{\lambda}\right)\right)\right)^{m \times m}, i \in \mathbf{Z}_{+}
$$

such that on $\Omega^{\prime \lambda}\left(r, C_{\lambda}\right)$ we have

(i) $\left|\partial_{x^{\prime}}^{\alpha^{\prime}} \partial_{\xi^{\prime}}^{\beta^{\prime}}\left(V_{i,(\mu, \nu)}^{\prime \prime \lambda}-\delta_{i, 0} \delta_{\mu, \nu}\right)\right|,\left|\partial_{x^{\prime}}^{\alpha^{\prime}} \partial_{\xi^{\prime}}^{\beta^{\prime}}\left(W_{i,(\mu, \nu)}^{\prime \prime \lambda}-\delta_{i, 0} \delta_{\mu, \nu}\right)\right|$

$$
\leq 100 m^{2} r^{2} \sum_{k \leq i}\left(i-k+\left|\alpha^{\prime}+\beta^{\prime}\right|\right) ! C_{\lambda}^{2 i-2 k+\frac{1}{4}\left|\alpha^{\prime}+\beta^{\prime}\right|}
$$

$\times\left(16 r^{m+2-\lambda}\right)^{k}\left(\operatorname{Im} \xi_{n}\right)^{-i+k-\left|\beta^{\prime}\right|}$,

(ii) $\left|\partial_{x^{\prime}}^{\alpha^{\prime}} \partial_{\xi^{\prime}}^{\beta^{\prime}}\left(\bar{M}_{i,(\mu, \nu)}^{\prime \prime \lambda}-\bar{L}_{i,(\mu, \nu)}^{\prime \prime}\right)\right|$

$\leq \sum_{k \leq i}\left(i-k+\left|\alpha^{\prime}+\beta^{\prime}\right|\right) ! C_{\lambda}^{2 i-2 k+\frac{1}{4}\left|\alpha^{\prime}+\beta^{\prime}\right|+1}\left(4 r^{m+2-\lambda}\right)^{k}\left(\operatorname{Im} \xi_{n}\right)^{-i+k-\left|\beta^{\prime}\right|}$

$\times\left(1-\delta_{i, 0}+\left|x_{1}\right|^{-q-1}\left(\operatorname{Im} \xi_{n}\right)^{-\frac{1}{m}}\right)$,

(iii) $V_{i,(\mu, \nu)}^{\prime \prime \lambda}, W_{i,(\mu, \nu)}^{\prime \prime \lambda}=\delta_{i, 0} \delta_{\mu, \nu}, \quad \mu \neq \lambda$ or $\nu \leq \lambda$,

(iv) $\bar{M}_{i,(\mu, \nu)}^{\prime \prime \lambda}=\delta_{i, 0} \delta_{\mu, \nu} \bar{L}_{0,(\mu, \nu)}^{\prime \prime}, \quad \mu \leq \lambda$ or $\nu \leq \lambda$,

(v) $V^{\prime \prime \lambda} \circ W^{\prime \prime \lambda}=W^{\prime \prime \lambda} \circ V^{\prime \prime \lambda}=I_{m}$,

(vi) $\partial_{x_{1}} V^{\prime \prime \lambda}+\bar{M}^{\prime \lambda} \circ V^{\prime \prime \lambda}-V^{\prime \prime \lambda} \bar{M}^{\prime \prime \lambda}=O$. 
The proof is almost the same as Lemma 5, and we only point out some differences. We first note that

$$
V^{\prime \prime \lambda, j}=\left(\begin{array}{cccccc}
1, & & & & & 0 \\
& \ddots & & & & \\
& & 1, & * & \cdots & * \\
& & & 1, & & 0 \\
0 & & & & & 1
\end{array}\right)(\lambda .
$$

If $\lambda+1 \leq \nu \leq m$, calculating the $(\lambda, \nu)$-component of (vi) in the statement we obtain

$$
\partial_{x_{1}} V_{(\lambda, \nu)}^{\prime \prime \lambda}+\sum_{1 \leq \kappa \leq m}\left(\bar{M}_{(\lambda, \kappa)}^{\prime \lambda} \circ V_{(\kappa, \nu)}^{\prime \prime \lambda}-V_{(\lambda, \kappa)}^{\prime \prime \lambda} \circ \bar{M}_{(\kappa, \nu)}^{\prime \prime \lambda}\right)=0
$$

If $\mu \geq \lambda+1, \nu \geq \lambda+1$, calculating the $(\mu, \nu)$-component of (vi) we obtain $\bar{M}_{(\mu, \nu)}^{\prime \lambda}=\bar{M}_{(\mu, \nu)}^{\prime \prime \lambda}$. The other components of the left hand side of (vi) always vanish. Therefore we need to solve $(27)$ and define $\bar{M}_{(\mu, \nu)}^{\prime \prime \lambda}=\bar{M}_{(\mu, \nu)}^{\prime \lambda}$ for $\mu \geq \lambda+1, \nu \geq \lambda+1$. We can rewrite (27) in the form

$$
\partial_{x_{1}} V_{(\lambda, \nu)}^{\prime \prime \lambda}+\bar{M}_{(\lambda, \nu)}^{\prime \lambda}+\bar{L}_{(\lambda, \lambda)}^{\prime \prime \lambda} \circ V_{(\lambda, \nu)}^{\prime \prime \lambda}-\sum_{\lambda+1 \leq \kappa \leq m} V_{(\lambda, \kappa)}^{\prime \prime \lambda} \circ \bar{M}_{(\kappa, \nu)}^{\prime \lambda}=0 .
$$

We can solve this equation by successive approximation.

$$
\partial_{x_{1}} V_{i,(\lambda, \nu)}^{\prime \prime \lambda, j}+\left(\bar{L}_{0,(\lambda, \lambda)}^{\prime \prime}-\bar{L}_{0,(\nu, \nu)}^{\prime \prime}\right) V_{i,(\lambda, \nu)}^{\prime \prime \lambda, j}=F_{i,(\lambda, \nu)}^{\prime \prime \lambda, j}=\sum_{0 \leq k \leq 4} F_{i,(\lambda, \nu)}^{\prime \prime \lambda, j, k},
$$

where

$$
\begin{aligned}
& F_{i,(\lambda, \nu)}^{\prime \prime \lambda, j, 0}=-\bar{M}_{i,(\lambda, \nu)}^{\prime \lambda}, \\
& F_{i,(\lambda, \nu)}^{\prime \prime \lambda, j, 1}=\sum_{\lambda+1 \leq \kappa \leq m} V_{i,(\lambda, \kappa)}^{\prime \prime \lambda, j-1}\left(\bar{M}_{0,(\kappa, \nu)}^{\prime \lambda}-\delta_{\kappa, \nu} \bar{L}_{0,(\nu, \nu)}^{\prime \prime}\right), \\
& F_{\substack{i,(\lambda, \nu) \\
\prime \prime \lambda, j, 2}}=\sum_{\substack{\lambda+1 \leq \kappa \leq m \\
i^{\prime \prime}+\left|\alpha^{\prime}\right|=i \\
i^{\prime \prime} \neq i}} \frac{1}{\alpha^{\prime} !} \partial_{\xi^{\prime}}^{\alpha^{\prime}} V_{i^{\prime \prime},(\lambda, \kappa)}^{\prime \prime \lambda} \partial_{x^{\prime}}^{\alpha^{\prime}} \bar{M}_{0,(\kappa, \nu)}^{\prime \lambda},
\end{aligned}
$$




$$
\begin{aligned}
& F_{\substack{i,(\lambda, \nu) \\
\prime \prime \lambda, j, 3}} \sum_{\substack{\lambda+1 \leq \kappa \leq m \\
i^{\prime}+i^{\prime \prime}+\left|\alpha^{\prime}\right|=i \\
i^{\prime} \neq 0}} \frac{1}{\alpha^{\prime} !} \partial_{\xi^{\prime}}^{\alpha^{\prime}} V_{i^{\prime \prime},(\lambda, \kappa)}^{\prime \prime \lambda} \partial_{x^{\prime}}^{\alpha^{\prime}} \bar{M}_{i^{\prime},(\kappa, \nu)}^{\prime \lambda},
\end{aligned}
$$

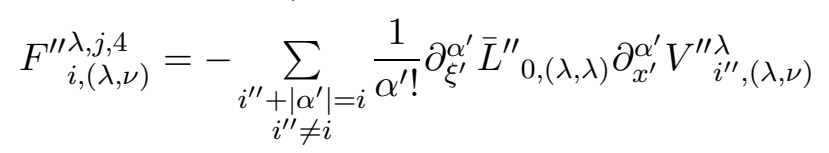

on $\Omega^{\prime \lambda}\left(r, C_{\lambda}\right)$. Here we define $V_{i,(\mu, \nu)}^{\prime \prime \lambda,-1}=0$. Let us define $\tilde{V}_{i,(\mu, \nu)}^{\prime \prime \lambda, j}, \tilde{F}_{i,(\mu, \nu)}^{\prime \prime \lambda, j}$ as before. This time we obtain a solution

$\tilde{V}_{i,(\lambda, \nu)}^{\prime \prime \lambda, j}\left(x, \xi^{\prime}\right)=\delta_{i, 0} \delta_{j, 0} \delta_{\lambda, \nu}+\int_{\delta \mp, \lambda\left(x_{1}\right)} \exp \left(\phi_{\lambda, \nu}\left(x, t, \xi^{\prime}\right)\right) \tilde{F}_{i,(\lambda, \nu)}^{\prime \prime \lambda, j}\left(t, x^{\prime}, \xi^{\prime}\right) d t$.

We can also define $V^{\prime \prime \lambda}=\lim _{j \rightarrow \infty} V^{\prime \prime \lambda}, j$, and the inverse asymptotic series $W^{\prime \prime \lambda}$ as before. In this way we can prove Lemma 6. We also obtain Proposition 3 by taking

$$
V^{\lambda}=V^{\lambda-1} \circ V^{\prime \lambda} \circ V^{\prime \prime \lambda}, W^{\lambda}=W^{\prime \prime \lambda} \circ W^{\prime \lambda} \circ W^{\lambda-1}, \quad \bar{M}^{\lambda}=\bar{M}^{\prime \prime \lambda} .
$$

We remind the reader that $U$ (resp. $V^{m}$ ) transforms $\bar{L}$ into $\bar{L}^{\prime \prime}$ (resp. $\bar{L}^{\prime \prime}$ into $\left.\bar{M}^{m}\right)$ on $\Omega^{\prime m}\left(r, C_{m}\right)$. We next want to transform $\bar{M}^{m}$ into $\bar{M}$. But this is very easy because they are diagonal matrices this time. Let $1<<a(<<1 / r)$. In this section we define

$$
\begin{aligned}
& \omega(a)=\left\{\left(x, \xi^{\prime}\right) \in \mathbf{C}^{n} \times \mathbf{C}^{n-1} ; a^{3}\left(\operatorname{Im} \xi_{n}\right)^{-1 / m q}<\left|x_{1}\right|<a^{-1}, a\left|x^{\prime}\right|<1\right. \\
& \left.\left|\arg x_{1}\right|<3 \pi, a\left|\xi^{\prime \prime \prime}\right|<\operatorname{Im} \xi_{n}, a\left|\operatorname{Re} \xi_{n}\right|<\operatorname{Im} \xi_{n}, a^{5 m q}<\operatorname{Im} \xi_{n}\right\}
\end{aligned}
$$

Note that we have $\omega(a) \supset \Omega^{\prime j}\left(r, C_{j}\right)$ for $1 \leq j \leq m$. Let $x_{1}^{o}=1 / 2 a$. If $\left(x, \xi^{\prime}\right) \in \omega(a)$, then we define

$\gamma_{0}\left(x_{1}\right)=\left\{s\left|x_{1}\right|+(1-s) x_{1}^{o} ; 0 \leq s \leq 1\right\} \cup\left\{\left|x_{1}\right| \exp \left(\sqrt{-1} s \arg x_{1}\right) ; 0 \leq s \leq 1\right\}$

It is easy to see that if $\left(x, \xi^{\prime}\right) \in \omega(a), t \in \gamma_{0}\left(x_{1}\right)$, then we have $\left(t, x^{\prime}, \xi^{\prime}\right) \in$ $\omega(a)$. We define $\bar{M}^{+}=\bar{M}^{-, m}=\bar{M}, \bar{M}^{-}=\bar{M}^{+, m}=\bar{M}^{m}$. Then we have the following

Lemma 7. There exist diagonal matrices $X_{i}^{ \pm} \in(\mathcal{O}(\omega(a)))^{m \times m}$ such that on $\omega($ a) we have 
(i) $\left|\partial_{x^{\prime}}^{\alpha^{\prime}} \partial_{\xi^{\prime}}^{\beta^{\prime}} X_{i,(\mu, \mu)}^{ \pm}\right| \leq \sum_{j \leq i} \frac{\left(i+\left|\alpha^{\prime}+\beta^{\prime}\right|\right) !}{j !} a^{\frac{i}{2}+\frac{1}{4}\left|\alpha^{\prime}+\beta^{\prime}\right|+1}$
$\quad \times\left(\rho_{0}\left(x_{1}\right)\right)^{j}\left(\operatorname{Im} \xi_{n}\right)^{-i+j-\left|\beta^{\prime}\right|} \exp \left\{a \rho_{0}\left(x_{1}\right)\left(\operatorname{Im} \xi_{n}\right)^{(m q-1) / m q}\right\}$,
(ii) $X^{ \pm} \circ X^{\mp}=I_{m}, \quad \partial_{x_{1}} X^{ \pm}+\bar{M}^{ \pm, m} X^{ \pm}-\bar{M}^{ \pm} \circ X^{ \pm}=O$.

Proof. Let $\bar{N}=\bar{M}-\bar{M}^{m}$. We need to solve

$$
\begin{aligned}
& \partial_{x_{1}} X_{i,(\mu, \mu)}^{ \pm} \mp \bar{N}_{0,(\mu, \mu)} X_{i,(\mu, \mu)}^{ \pm}=G_{i,(\mu, \mu)}^{ \pm}, \\
& G_{i,(\mu, \mu)}^{ \pm}=-\sum_{\substack{i^{\prime}+i^{\prime \prime}+\left|\alpha^{\prime}\right|=i \\
i^{\prime \prime} \neq i}} \frac{1}{\alpha^{\prime} !}\left(\partial_{\xi^{\prime}}^{\alpha^{\prime}} \bar{M}_{i^{\prime},(\mu, \mu)}^{ \pm, m} \partial_{x^{\prime}}^{\alpha^{\prime}} X_{i^{\prime \prime},(\mu, \mu)}^{ \pm}-\partial_{\xi^{\prime}}^{\alpha^{\prime}} X_{i^{\prime \prime},(\mu, \mu)}^{ \pm} \partial_{\xi^{\prime}}^{\alpha^{\prime}} \bar{M}_{(\mu, \mu)}^{ \pm}\right), \\
& X_{i,(\mu, \mu)}^{ \pm}\left(x_{1}^{o}, x^{\prime}, \xi^{\prime}\right)=\delta_{i, 0}
\end{aligned}
$$

for each $i \in \mathbf{Z}_{+}$. Since we have $\left|\bar{N}_{0,(\mu, \mu)}\right| \leq \sqrt{a}\left(\operatorname{Im} \xi_{n}\right)^{(m q-1) / m q}$, we have (i) for $X_{0,(\mu, \mu)}^{ \pm}\left(x, \xi^{\prime}\right)=\exp \left\{ \pm \int_{\delta_{0}\left(x_{1}\right)} \bar{N}_{0,(\mu, \mu)}\left(t, x^{\prime}, \xi^{\prime}\right) d t\right\}$. Let $i_{0} \geq 1$, and assume that (i) is true if $1 \leq i \leq i_{0}-1$. Since $\bar{M}_{(\mu, \mu)}^{m}=\bar{L}_{0,(\mu, \mu)}^{\prime \prime}$, we have

$$
\begin{aligned}
& \left|\partial_{x^{\prime}}^{\alpha^{\prime}} \partial_{\xi^{\prime}}^{\beta^{\prime}} \bar{M}_{(\mu, \mu)}^{ \pm, m}\left(x, \xi^{\prime}\right)\right|,\left|\partial_{x^{\prime}}^{\alpha^{\prime}} \partial_{\xi^{\prime}}^{\beta^{\prime}} \bar{M}_{(\mu, \mu)}^{ \pm}\left(x, \xi^{\prime}\right)\right| \\
\leq & \alpha^{\prime} ! \beta^{\prime} ! a^{\frac{1}{10}\left(\left|\alpha^{\prime}+\beta^{\prime}\right|+1\right)}\left(\operatorname{Im} \xi_{n}\right)^{1-\left|\beta^{\prime}\right|} .
\end{aligned}
$$

It follows that

$$
\begin{aligned}
&\left|\partial_{x^{\prime}}^{\alpha^{\prime}} \partial_{\xi^{\prime}}^{\beta^{\prime}} X_{i,(\mu, \mu)}^{ \pm}\right| \leq \sum_{(29)} \frac{\alpha^{\prime} ! \beta^{\prime} !}{\alpha^{\prime(1)} ! \alpha^{\prime(2)} ! \alpha^{\prime(3) !} ! \beta^{\prime(1)} ! \beta^{\prime(2)} ! \beta^{\prime(3)} ! \gamma^{\prime} !} \\
& \times \int_{\delta_{0}\left(x_{1}\right)}\left|\partial_{x^{\prime}}^{\alpha^{\prime(1)}} \partial_{\xi^{\prime}}^{\beta^{\prime(1)}} \exp \left( \pm \int_{\delta_{0}\left(x_{1}\right)-\delta_{0}(t)} \bar{N}_{0,(\mu, \mu)}\left(s, x^{\prime}, \xi^{\prime}\right) d s\right)\right| \\
& \times\left\{\left|\partial_{x^{\prime}}^{\alpha^{\prime(2)}} \partial_{\xi^{\prime}}^{\beta^{\prime(2)}+\gamma^{\prime}} \bar{M}_{(\mu, \mu)}^{ \pm, m}\left(t, x^{\prime}, \xi^{\prime}\right) \partial_{x^{\prime}}^{\alpha^{\prime(3)}+\gamma^{\prime}} \partial_{\xi^{\prime}}^{\beta^{\prime(3)}} X_{i^{\prime \prime},(\mu, \mu)}^{ \pm}\left(t, x^{\prime}, \xi^{\prime}\right)\right|\right. \\
&\left.+\left|\partial_{x^{\prime}}^{\alpha^{\prime(3)}} \partial_{\xi^{\prime}}^{\beta^{\prime(3)}+\gamma^{\prime}} X_{i^{\prime \prime},(\mu, \mu)}^{ \pm}\left(t, x^{\prime}, \xi^{\prime}\right) \partial_{x^{\prime}}^{\alpha^{\prime(2)}+\gamma^{\prime}} \partial_{\xi^{\prime}}^{\beta^{\prime \prime(2)}} \bar{M}_{(\mu, \mu)}^{ \pm}\left(t, x^{\prime}, \xi^{\prime}\right)\right|\right\}|d t|,
\end{aligned}
$$

where the summation is taken for

$$
i^{\prime \prime}+\left|\gamma^{\prime}\right|=i, i^{\prime \prime} \neq i, \alpha^{(1)}+\alpha^{(2)}+\alpha^{\prime(3)}=\alpha^{\prime}, \beta^{\prime(1)}+\beta^{\prime(2)}+\beta^{\prime(3)}=\beta^{\prime} .
$$


Using (28) and the assumption for $0 \leq i^{\prime \prime} \leq i_{0}-1$, it follows that

$$
\begin{aligned}
& \left|\partial_{x^{\prime}}^{\alpha^{\prime}} \partial_{\xi^{\prime}}^{\beta^{\prime}} X_{i,(\mu, \mu)}^{ \pm}\right| \leq \sum_{(29)} \sum_{j^{\prime \prime} \leq i^{\prime \prime}} 2 a^{-\frac{1}{20}\left(\left|\alpha^{\prime(1)}+\alpha^{\prime(2)}+\beta^{\prime(1)}+\beta^{\prime(2)}+\gamma^{\prime}\right|\right)} \frac{\left(i+\left|\alpha^{\prime}+\beta^{\prime}\right|\right) !}{\left(j^{\prime \prime}+1\right) !} \\
\times & a^{\frac{i}{2}+\frac{1}{4}\left|\alpha^{\prime}+\beta^{\prime}\right|+1}\left(\rho_{0}(t)\right)^{j^{\prime \prime}+1}\left(\operatorname{Im} \xi_{n}\right)^{1-i+j^{\prime \prime}-\left|\beta^{\prime}\right|} \exp \left\{a \rho_{0}\left(x_{1}\right)\left(\operatorname{Im} \xi_{n}\right)^{(m q-1) / m q}\right\}
\end{aligned}
$$

Let $j=j^{\prime \prime}+1$. Since $j^{\prime \prime} \leq i^{\prime \prime} \leq i-1$, we obtain Lemma 7 .

Let $C>>C_{m}$. We define $Y^{+}=U^{+} \circ V^{m} \circ X^{+}, Y^{-}=X^{-} \circ W^{m} \circ U^{-}$. Using the notation in section $3, Y^{ \pm}$are defined on $\Omega_{0}^{\prime \theta}(C)$ for each $\theta$, so we denote them $Y^{ \pm, \theta}$ (Precisely speaking, the number $C$ and the symbol $\bar{M}^{m}$ depend on the choice of $\theta$ ). Finally we need to calculate $Y^{ \pm, \theta}$ also on $\Omega^{\prime \prime}{ }_{0}(C)$, but this is easy because $x_{1}$ is very small on this domain. We define $x_{1}^{(k)}=\left(3 / 4 C^{5}\right)(k+2)^{-1 / m q} \exp (\sqrt{-1} \theta)$ for each $k \in \mathbf{Z}_{+}$. It is easy to see that if $\left(x, \xi^{\prime}\right) \in \Omega^{\prime \theta}{ }_{k}(C) \backslash \Omega^{\prime \theta}{ }_{k+2}(C)$, then we have $\left(x_{1}^{(k)}, x^{\prime}, \xi^{\prime}\right) \in\left(\Omega^{\prime \prime}{ }_{k}(C) \backslash\right.$ $\left.\Omega^{\prime \prime}{ }_{k+2}(C)\right) \cap \Omega^{\prime \theta}(C)$. Now we have the following

LEMMA 8. Let $\sigma_{+}(\mu, \nu)=(m-1) \mu / m$ and $\sigma_{-}(\mu, \nu)=-(m-1) \nu / m$.

(i) We have

$$
\begin{gathered}
\quad\left|\partial_{x^{\prime}}^{\alpha^{\prime}} \partial_{\xi^{\prime}}^{\beta^{\prime}} Y_{i,(\mu, \nu)}^{ \pm, \theta}\right| \leq \sum_{j \leq i}\left(i-j+\left|\alpha^{\prime}+\beta^{\prime}\right|\right) ! C^{i-j+\frac{1}{4}\left|\alpha^{\prime}+\beta^{\prime}\right|+1} \\
\times a^{-\frac{j}{6}}\left(\operatorname{Im} \xi_{n}\right)^{-i+j-\left|\beta^{\prime}\right|+\sigma_{ \pm}(\mu, \nu)} \exp \left\{C\left(\operatorname{Im} \xi_{n}\right)^{(m q-1) / m q}\right\}
\end{gathered}
$$

on $\Omega_{0}^{\prime \theta}(C)$.

(ii) We have

$$
\begin{aligned}
& \quad\left|\partial_{x^{\prime}}^{\alpha^{\prime}} \partial_{\xi^{\prime}}^{\beta^{\prime}} Y_{i,(\mu, \nu)}^{ \pm, \theta}\right| \leq \sum_{j \leq i}\left(i-j+\left|\alpha^{\prime}+\beta^{\prime}\right|\right) ! 2 C^{2 i-2 j+\frac{1}{4}\left|\alpha^{\prime}+\beta^{\prime}\right|+1} a^{-\frac{j}{6}} \\
& \times\left(\operatorname{Im} \xi_{n}\right)^{-i+j-\left|\beta^{\prime}\right|+\sigma_{ \pm}(\mu, \nu)} \exp \left\{C^{3}\left(\operatorname{Im} \xi_{n}\right)^{(m q-1) / m q}+C^{4}\left|x_{1}-x_{1}^{(k)}\right| \operatorname{Im} \xi_{n}\right\} \\
& \text { on } \Omega_{k}^{\prime \prime}(C) \backslash \Omega_{k+2}^{\prime \prime}(C) .
\end{aligned}
$$

Proof. (i) is already proved. We prove (ii). Let $\bar{L}^{+}=\bar{M}^{-}=\bar{L}, \bar{L}^{-}=$ $\bar{M}^{+}=\bar{M}$. We have $\partial_{x_{1}} Y^{ \pm, \theta}+\bar{L}^{ \pm} \circ Y^{ \pm, \theta}-Y^{\theta, \pm} \circ \bar{M}^{ \pm}=O$ on $\Omega_{k}^{\prime \theta}(C)$. We 
can uniquely extend $Y^{ \pm, \theta}$ on $\Omega^{\prime \prime}{ }_{k}(C) \backslash \Omega^{\prime \prime}{ }_{k+2}(C)$ by this equation and we can write as $Y_{i}^{ \pm, \theta}=\sum_{j \in \mathbf{Z}_{+}} Y_{i}^{ \pm, \theta, j}$, where

$$
\begin{aligned}
& Y_{i,(\mu, \nu)}^{ \pm, \theta, j}\left(x, \xi^{\prime}\right)=\delta_{j, 0} Y_{i,(\mu, \nu)}^{ \pm, \theta}\left(x_{1}^{(k)}, x^{\prime}, \xi^{\prime}\right) \\
& -\sum_{\substack{i^{\prime}+i^{\prime \prime}+\left|\alpha^{\prime}\right|=i \\
1 \leq \kappa \leq m}} \frac{1}{\alpha^{\prime} !} \int_{x_{1}^{(k)}}^{x_{1}}\left\{\partial_{\xi^{\prime}}^{\alpha^{\prime}} \bar{L}_{i^{\prime},(\mu, \kappa)}^{ \pm}\left(t, x^{\prime}, \xi^{\prime}\right) \partial_{x^{\prime}}^{\alpha^{\prime}} Y_{i^{\prime \prime},(\kappa, \nu)}^{ \pm, \theta, j-1}\left(t, x^{\prime}, \xi^{\prime}\right)\right. \\
& \left.-\partial_{\xi^{\prime}}^{\alpha^{\prime}} Y_{i^{\prime \prime},(\mu, \kappa)}^{ \pm, \theta, j-1}\left(t, x^{\prime}, \xi^{\prime}\right) \partial_{x^{\prime}}^{\alpha^{\prime}} \bar{M}_{i^{\prime},(\kappa, \nu)}^{ \pm}\left(t, x^{\prime}, \xi^{\prime}\right)\right\} d t
\end{aligned}
$$

$\left(\right.$ Here $\left.Y^{ \pm, \theta,-1}=O\right)$. We can prove that

$$
\begin{aligned}
& \left|\partial_{x^{\prime}}^{\alpha^{\prime}} \partial_{\xi^{\prime}}^{\beta^{\prime}} Y_{i,(\mu, \nu)}^{ \pm, \theta, j}\right| \leq 2^{-j} \sum_{\ell \leq i}\left(i-\ell+\left|\alpha^{\prime}+\beta^{\prime}\right|\right) ! \\
\times & C^{2 i-2 \ell+\frac{1}{4}\left|\alpha^{\prime}+\beta^{\prime}\right|+1} a^{-\frac{\ell}{6}}\left(\operatorname{Im} \xi_{n}\right)^{-i+\ell-\left|\beta^{\prime}\right|+\sigma_{ \pm}(\mu, \nu)} \\
\times & \exp \left\{C^{3}\left(\operatorname{Im} \xi_{n}\right)^{(m q-1) / m q}+C^{4}\left|x_{1}-x_{1}^{(k)}\right| \operatorname{Im} \xi_{n}\right\}
\end{aligned}
$$

on $\Omega^{\prime \prime}{ }_{k}(C) \backslash \Omega^{\prime \prime}{ }_{k+2}(C)$. If $j=0,(31)$ is a direct consequence of (30). If $j \geq 1$, we can easily prove (31) by induction on $j$. Summing up (31) for $j \in \mathbf{Z}_{+}$, we obtain (ii).

Proof of Proposition 2. Let $\left(x, \xi^{\prime}\right) \in \Omega^{\prime \prime}{ }_{0}(C)$. Then we have $\left(x, \xi^{\prime}\right) \in \Omega^{\prime \prime}{ }_{k}(C) \backslash \Omega^{\prime \prime}{ }_{k+2}(C)$ for some $k \in \mathbf{Z}_{+}$. We have

$$
\begin{aligned}
& \left|x_{1}-x_{1}^{(k)}\right| \leq\left(3 / 4 C^{5}\right)(k+2)^{-1 / m q}+C^{-1}\left(\operatorname{Im} \xi_{n}\right)^{-1 / 2 m q} \\
\leq & 2 C^{-1}\left(\operatorname{Im} \xi_{n}\right)^{-1 / 2 m q}
\end{aligned}
$$

on $\Omega^{\prime \prime}{ }_{k}(C) \backslash \Omega^{\prime \prime}{ }_{k+2}(C)$. From Lemma 8 it follows that

$$
\begin{aligned}
& \left|Y_{i,(\mu, \nu)}^{ \pm, \theta}\right| \leq \sum_{j \leq i}(i-j) ! 2 C^{2 i-2 j+1} a^{-\frac{j}{6}}\left(\operatorname{Im} \xi_{n}\right)^{-i+j+\sigma_{ \pm}(\mu, \nu)} \\
\times & \exp \left\{2 C^{3}\left(\operatorname{Im} \xi_{n}\right)^{(2 m q-1) / 2 m q}\right\}
\end{aligned}
$$

on $\Omega_{0}^{\theta}(C)$. Since $(i-j) !\left(\operatorname{Im} \xi_{n}\right)^{-i+j} \leq C^{-3 i+3 j}$ for $0 \leq j \leq i$ on $\Omega_{i}^{\theta}(C)$, we have

$$
\left|Y_{i,(\mu, \nu)}^{ \pm, \theta}\right| \leq 4 C a^{-\frac{i}{6}} \exp \left(C^{4}\left(\operatorname{Im} \xi_{n}\right)^{(2 m q-1) / 2 m q}\right)
$$

on $\Omega_{i}^{\theta}(C)$. 


\section{Calculation of Stokes Operators}

In this section we prove Theorem 2. We assume that $j_{0} \in J$ satisfies (10) (The case (9) is similar). We may assume $j_{0}=1$ and then from (11) it follows that $q_{1}=1$. In Proposition 1 we may choose an arbitrary $\theta \in[0,2 \pi]$, and we may choose $\theta^{\prime 1}, \theta^{\prime \prime 1}$ arbitrarily as far as they satisfy $\theta^{\prime 1}<\theta<\theta^{\prime \prime 1}, \pi / 2<\theta^{\prime \prime 1}-\theta^{\prime 1}<2 \pi / 3$. Note that for such $\theta^{\prime 1}$ and $\theta^{\prime \prime 1}$, we can always complement some $\theta^{\prime j}, \theta^{\prime \prime j}, 2 \leq j \leq m$. Let us consider the following three cases:

$$
\begin{array}{lll}
\theta=0, & \theta^{\prime 1}=-7 \pi / 24, & \theta^{\prime \prime 1}=7 \pi / 24 \\
\theta=\pi / 2, & \theta^{\prime 1}=5 \pi / 24, & \theta^{\prime \prime 1}=19 \pi / 24 \\
\theta=\pi, & \theta^{\prime 1}=17 \pi / 24, & \theta^{\prime \prime 1}=31 \pi / 24 .
\end{array}
$$

In each case, it follows that

$$
\begin{aligned}
& k_{1}=0, \quad \theta^{-, 1}=-\pi / 4, \quad \theta^{+, 1}=\pi / 4, \\
& k_{1}=1, \quad \theta^{-, 1}=3 \pi / 4, \quad \theta^{+, 1}=\pi / 4, \\
& k_{1}=2, \quad \theta^{-, 1}=3 \pi / 4, \quad \theta^{+, 1}=5 \pi / 4 \text {, }
\end{aligned}
$$

respectively. In any case of (32.l), we may choose common constants $r, C_{1}, \cdots, C_{m}$, and calculate $V^{m}, W^{m}$. To distinguish them each other, we denote each of them by $V^{m,[\ell]}, W^{m,[\ell]}$. Let $C>>C_{m}$. These asymptotic series are defined on $\Omega^{\prime m}(r, C)$ for the corresponding $\theta, \theta^{\prime m}, \theta^{\prime \prime m}$, and we denote each of these domains by $\Omega^{\prime m},[\ell](r, C)$. We remind the reader that we have

$$
\begin{aligned}
& V^{m,[\ell]}=V^{\prime 1,[\ell]} \circ V^{\prime \prime 1,[\ell]} \circ V^{\prime 2,[\ell]} \circ V^{\prime \prime 2,[\ell]} \circ \cdots \circ V^{\prime m,[\ell]} \circ V^{\prime \prime m,[\ell]}, \\
& W^{m,[\ell]}=W^{\prime \prime m,[\ell]} \circ W^{\prime m,[\ell]} \circ \cdots \circ W^{\prime \prime 2,[\ell]} \circ W^{\prime 2,[\ell]} \circ W^{1,[\ell]} .
\end{aligned}
$$

In the proof of Proposition 3 we have $V_{(\mu, 1)}^{\prime j,[\ell]}=\delta_{\mu, 1}$ for $2 \leq j \leq m, V_{(\mu, 1)}^{\prime \prime j,[\ell]}=$ $\delta_{\mu, 1}$ for $1 \leq j \leq m$, and thus $V_{(\mu, 1)}^{m,[\ell]}=V_{(\mu, 1)}^{\prime 1,[\ell]}$ for $1 \leq \mu \leq m$. Similarly we have $W_{(1, \nu)}^{m,[\ell]}=W_{(1, \nu)}^{1,[\ell]}$ for $1 \leq \nu \leq m$. Therefore we have $V_{(\mu, 1)}^{m,[\ell]}, W_{(1, \nu)}^{m,[\ell]} \in$ $\Omega^{\prime 1,[\ell]}(r, C)$. Let us compare $V_{(\mu, 1)}^{m,[2]}\left(=V_{(\mu, 1)}^{\prime 1,[2]}\right)$ and $V_{(\mu, 1)}^{m,[3]}\left(=V_{(\mu, 1)}^{\prime 1,[3]}\right)$. They 
are calculated by integration along $\delta^{-, 1}\left(x_{1}\right)$, where the path $\delta^{-, 1}\left(x_{1}\right)$ is a curve from $x_{1}^{-, 1}$ to $x_{1}$, which depends on the choice of $\theta^{-, 1}$. However, in (32.2) and (32.3) we use the same $\theta^{-, 1}$ (and thus $\gamma^{-, 1}\left(x_{1}\right)$ ). Therefore we have $V_{(\mu, 1)}^{m,[2]}=V_{(\mu, 1)}^{m,[3]}$, and they define a symbol on $\Omega^{\prime 1,[2]}(r, C) \cup \Omega^{\prime 1,[3]}(r, C)$.

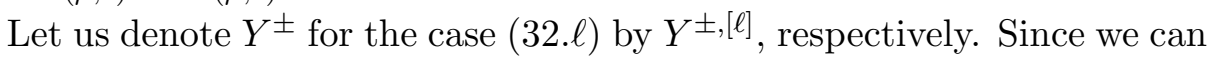
take common symbols $U, U^{\prime}, X^{ \pm}$for any $\ell$ and $X^{ \pm}$are diagonal matrices, we have

$$
\begin{aligned}
& \left(Y^{-,[k]}\left(0, x^{\prime}, \xi^{\prime}\right) \circ Y^{+,[\ell]}\left(0, x^{\prime}, \xi^{\prime}\right)\right)_{(1,1)} \\
= & \left(X^{-} \circ W^{m,[k]} \circ U^{\prime} \circ U \circ V^{m,[\ell]} \circ X^{+}\right)_{(1,1)}\left(0, x^{\prime}, \xi^{\prime}\right) \\
= & \sum_{1 \leq \kappa \leq m} X_{(1,1)}^{-}\left(0, x^{\prime}, \xi^{\prime}\right) \circ W_{(1, \kappa)}^{1,[k]}\left(0, x^{\prime}, \xi^{\prime}\right) \circ V_{(\kappa, 1)}^{\prime 1,[\ell]}\left(0, x^{\prime}, \xi^{\prime}\right) \circ X_{(1,1)}^{+}\left(0, x^{\prime}, \xi^{\prime}\right) .
\end{aligned}
$$

$Z_{+}\left(x^{\prime}, \xi^{\prime}\right)$ (resp. $Z_{-}\left(x^{\prime}, \xi^{\prime}\right)$ ) is calculated by considering $\theta=0$ (resp. $\theta=\pi)$, and we have $Z_{+}\left(x^{\prime}, \xi^{\prime}\right)=Y^{-,[1]}\left(0, x^{\prime}, \xi^{\prime}\right)\left(\operatorname{resp} .\left(Z_{-}\right)^{-1}\left(x^{\prime}, \xi^{\prime}\right)=\right.$ $\left.Y^{+,[3]}\left(0, x^{\prime}, \xi^{\prime}\right)\right)$. It follows that

$$
\begin{aligned}
& Z_{+-,(1,1)}\left(x^{\prime}, \xi^{\prime}\right)=\left(Z_{+} \circ\left(Z_{-}\right)^{-1}\right)_{(1,1)}\left(x^{\prime}, \xi^{\prime}\right) \\
= & \sum_{1 \leq \kappa \leq m} X_{(1,1)}^{-}\left(0, x^{\prime}, \xi^{\prime}\right) \circ W_{(1, \kappa)}^{1,[1]}\left(0, x^{\prime}, \xi^{\prime}\right) \circ V_{(\kappa, 1)}^{1,[3]}\left(0, x^{\prime}, \xi^{\prime}\right) \circ X_{(1,1)}^{+}\left(0, x^{\prime}, \xi^{\prime}\right) \\
= & \sum_{1 \leq \kappa \leq m} X_{(1,1)}^{-}\left(0, x^{\prime}, \xi^{\prime}\right) \circ W_{(1, \kappa)}^{1,[1]}\left(0, x^{\prime}, \xi^{\prime}\right) \circ V_{(\kappa, 1)}^{1,[2]}\left(0, x^{\prime}, \xi^{\prime}\right) \circ X_{(1,1)}^{+}\left(0, x^{\prime}, \xi^{\prime}\right)
\end{aligned}
$$

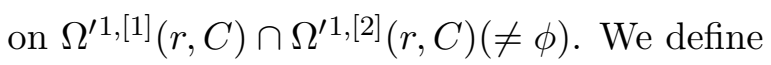

$$
\begin{aligned}
& V^{[1,2, \kappa]}\left(x, \xi^{\prime}\right)=W_{(1, \kappa)}^{1,[1]}\left(x, \xi^{\prime}\right) \circ V_{(\kappa, 1)}^{\prime 1,[2]}\left(x, \xi^{\prime}\right), \\
& V^{[1,2]}\left(x, \xi^{\prime}\right)=\sum_{1 \leq \kappa \leq m} V^{[1,2, \kappa]}, \\
& Y^{[1,2]}\left(x, \xi^{\prime}\right)=X_{(1,1)}^{-}\left(x, \xi^{\prime}\right) \circ V^{[1,2]}\left(x, \xi^{\prime}\right) \circ X_{(1,1)}^{+}\left(x, \xi^{\prime}\right) .
\end{aligned}
$$

Let $\left(x, \xi^{\prime}\right) \in \mathbf{C}^{n} \times \mathbf{C}^{n-1}$ satisfy

$$
\left\{\begin{array}{l}
C^{-3}<\left|x_{1}\right|<C^{-2}, C\left|\arg x_{1}-(\pi / 4)\right|<1, C\left|x^{\prime}\right|<1, \\
C\left|\xi^{\prime \prime \prime}\right|<\operatorname{Im} \xi_{n}, C\left|\operatorname{Re} \xi_{n}\right|<\operatorname{Im} \xi_{n}, C^{5 m q}(i+1)<\operatorname{Im} \xi_{n} .
\end{array}\right.
$$


Then we have $\left(x, \xi^{\prime}\right) \in \Omega^{\prime 1,[1]}(r, C) \cap \Omega^{\prime 1,[2]}(r, C)$. From Proposition 3 it is easy to see that $\left|V_{i}^{[1,2, \kappa]}-\delta_{i, 0} \delta_{\kappa, 1}\right| \leq r^{i+1}$. It follows that if

$$
\left\{\begin{array}{l}
C^{-3}<\left|x_{1}\right|<C^{-2}, C\left|\arg x_{1}-(\pi / 4)\right|<1, C^{2}\left|x^{\prime}\right|<1 \\
C^{2}\left|\xi^{\prime \prime \prime}\right|<\operatorname{Im} \xi_{n}, C^{2}\left|\operatorname{Re} \xi_{n}\right|<\operatorname{Im} \xi_{n}, C^{10 m q}(i+1)<\operatorname{Im} \xi_{n}
\end{array}\right.
$$

then $W_{0}^{[1,2]}=1 / V_{0}^{[1,2]}$ is well-defined, and we have

$$
\begin{aligned}
& \left|\partial_{x^{\prime}}^{\alpha^{\prime}} \partial_{\xi^{\prime}}^{\beta^{\prime}}\left(V_{i}^{[1,2]}-\delta_{i, 0}\right)\right| \leq m r^{i+1} C^{2\left|\alpha^{\prime}+\beta^{\prime}\right|} \alpha^{\prime} ! \beta^{\prime} !\left(\operatorname{Im} \xi_{n}\right)^{-\left|\beta^{\prime}\right|} \\
& \left|\partial_{x^{\prime}}^{\alpha^{\prime}} \partial_{\xi^{\prime}}^{\beta^{\prime}}\left(W_{0}^{[1,2]}-1\right)\right| \leq 2 m r C^{2\left|\alpha^{\prime}+\beta^{\prime}\right|} \alpha^{\prime} ! \beta^{\prime} !\left(\operatorname{Im} \xi_{n}\right)^{-\left|\beta^{\prime}\right|} .
\end{aligned}
$$

We define

$$
W_{i}^{[1,2]}=-\sum_{\substack{i^{\prime}+i^{\prime \prime}+\left|\alpha^{\prime}\right|=i \\ i^{\prime \prime} \neq i}} \frac{1}{\alpha^{\prime !}} W_{0}^{[1,2]} \partial_{\xi^{\prime}}^{\alpha^{\prime}} W_{i^{\prime \prime}}^{[1,2]} \partial_{x^{\prime}}^{\alpha^{\prime}} V_{i^{\prime}}^{[1,2]}, i \in \mathbf{N}
$$

It is defined on the domain $(35 . \mathrm{i})$, satisfies $\left(W^{[1,2]} \circ V^{[1,2]}\right)_{i}=\left(V^{[1,2]} \circ\right.$ $\left.W^{[1,2]}\right)_{i}=\delta_{i, 0}$ there, and on the domain (35.j) with $j=i+\left|\alpha^{\prime}\right|$ we have

$$
\left|\partial_{\xi^{\prime}}^{\alpha^{\prime}}\left(W_{i}^{[1,2]}-\delta_{i, 0}\right)\right| \leq 2 m r^{\frac{1}{2}(i+1)} C^{3\left|\alpha^{\prime}\right|} \alpha^{\prime} !\left(\operatorname{Im} \xi_{n}\right)^{-\left|\alpha^{\prime}\right|} .
$$

Furthermore, if (33.i), then we have

$$
\left|\partial_{x^{\prime}}^{\alpha^{\prime}} \partial_{\xi^{\prime}}^{\beta^{\prime}} X_{i,(1,1)}^{ \pm}\right| \leq a^{-\frac{i}{2}+1} C^{3\left|\alpha^{\prime}+\beta^{\prime}\right|} \alpha^{\prime} ! \beta^{\prime} !\left(\operatorname{Im} \xi_{n}\right)^{-\left|\beta^{\prime}\right|} \exp \left(C\left(\operatorname{Im} \xi_{n}\right)^{(m q-1) / m q}\right) .
$$

Therefore we obtain the following

\section{Proposition 4. If}

$$
\left\{\begin{array}{l}
C^{-3}<\left|x_{1}\right|<C^{-2}, C\left|\arg x_{1}-(\pi / 4)\right|<1 \\
C^{5}\left|\xi^{\prime \prime \prime}\right|<1, C^{5}\left|\operatorname{Re} \xi_{n}\right|<\operatorname{Im} \xi_{n}, \operatorname{Im} \xi_{n}>C^{25 m q}(i+1),
\end{array}\right.
$$

then there exists some holomorphic function $Y_{i}^{\prime[1,2]}$ such that

$$
\text { (i) }\left|\partial_{x^{\prime}}^{\alpha^{\prime}} \partial_{\xi^{\prime}}^{\beta^{\prime}} Y_{i}^{[1,2]}\right|,\left|\partial_{x^{\prime}}^{\alpha^{\prime}} \partial_{\xi^{\prime}}^{\beta^{\prime}} Y_{i}^{\prime[1,2]}\right|
$$




$$
\begin{aligned}
& \leq a^{-\frac{i}{20}} C^{5\left|\alpha^{\prime}+\beta^{\prime}\right|+5} \alpha^{\prime} ! \beta^{\prime} !\left(\operatorname{Im} \xi_{n}\right)^{-\left|\beta^{\prime}\right|} \exp \left(C^{5}\left(\operatorname{Im} \xi_{n}\right)^{(m q-1) / m q}\right), \\
& \text { (ii) } \quad\left(Y^{[1,2]} \circ Y^{\prime[1,2]}\right)_{i}=\left(Y^{\prime[1,2]} \circ Y^{[1,2]}\right)_{i}=\delta_{i, 0}, \\
& \text { (iii) } \partial_{x_{1}} Y_{i}^{[1,2]}+\left(\left(x_{1}^{q_{1}} a_{1}\right) \circ Y^{[1,2]}\right)_{i}-\left(Y^{[1,2]} \circ\left(x_{1}^{q_{1}} a_{1}\right)\right)_{i}=0, \\
& \text { (iv) } \partial_{x_{1}} Y_{i}^{\prime[1,2]}+\left(\left(x_{1}^{q_{1}} a_{1}\right) \circ Y^{\prime[1,2]}\right)_{i}-\left(Y^{\prime[1,2]} \circ\left(x_{1}^{q_{1}} a_{1}\right)\right)_{i}=0 .
\end{aligned}
$$

The proof is easy, and we only sketch it. We can solve (iii) and (iv) on

(36.i) $\left|x_{1}\right|<C^{-2}, C^{5}\left|\xi^{\prime \prime \prime}\right|<1, C^{5} \operatorname{Re} \xi_{n}<\operatorname{Im} \xi_{n}, \operatorname{Im} \xi_{n}>C^{25 m q}(i+1)$.

For instance, we can rewrite (iii) as

$$
\partial_{x_{1}} Y_{i}^{[1,2]}+\sum_{\substack{i^{\prime \prime}+\left|\alpha^{\prime}\right|=i \\ i^{\prime \prime} \neq i}} \frac{1}{\alpha^{\prime}}\left\{\partial_{\xi^{\prime}}^{\alpha^{\prime}}\left(x_{1}^{q_{1}} a_{1}\right) \partial_{x^{\prime}}^{\alpha^{\prime}} Y_{i^{\prime \prime}}^{[1,2]}-\partial_{\xi^{\prime}}^{\alpha^{\prime}} Y_{i^{\prime \prime}}^{[1,2]} \partial_{x^{\prime}}^{\alpha^{\prime}}\left(x_{1}^{q_{1}} a_{1}\right)\right\}=0
$$

By induction on $i$, it is easy to see that we can extend $Y_{i}^{[1,2]}, Y_{i}^{\prime[1,2]}$ to (36.i) and we have (i) there. Since $Z_{+-,(1,1)}\left(x^{\prime}, \xi^{\prime}\right)=Y^{[1,2]}\left(0, x^{\prime}, \xi^{\prime}\right)$, we obtain also Theorem 2 .

\section{References}

[1] Alinhac, S., Branching of singularities for a class of hyperbolic operators, Indiana Univ. Math. J. 27 (1978), 1027-1037.

[2] Amano, K., Branching of singularities for degenerate hyperbolic operators and Stokes phenomena, Proc. Japan Acad. 56 (1980), 206-209.

[3] Aoki, T., Symbols and formal symbols of pseudodifferential operators, Advanced Studies in Pure Mathematics 4 (1984), 181-208.

[4] Bove, A., Lewis, J. E. and C. Parenti, Propagation of singularities for Fuchsian operators, Lecture Notes in Math., vol. 984, Springer, Berlin-HeidelbergNew York, 1983.

[5] Cicognani, M. and L. Zanghirati, Propagation of analytic and Gevrey singularities for operators with non-involutive characteristics, J. Math. Kyoto Univ. 33 (1989), 633-646.

[6] Hanges, N., Parametrices and propagation of singularities for operators with non-involutive characteristics, Indiana Univ. Math. J. 28 (1979), 87-97.

[7] Hörmander, L., Fourier integral operators I, Acta Math. 127 (1971), 79-183. 
[8] Iwano, M. and Y. Shibuya, Reduction of the order of a linear ordinary differential equation containing a small parameter, Kōdai Math. Report 15 (1963), $1-28$.

[9] Kashiwara, M. and T. Kawai, Microhyperbolic pseudodifferential operators I, J. Math. Soc. Japan 27 (1975), 359-404.

[10] Nakane, S., Propagation of singularities and uniqueness in the Cauchy problem at a class of doubly characteristic points, Comm. Partial Differential Equations 6 (1981), 917-927.

[11] Nishitani, T., Hyperbolic operators with symplectic multiple characteristics, J. Math. Kyoto Univ. 29 (1989), 405-447.

[12] Ôaku, T., A canonical form of a system of microdifferential equations with non-involutory characteristics and branching of singularities, Invent. Math. 65 (1982), 491-525.

[13] Sato, M., Kawai, T. and M. Kashiwara, Microfunctions and pseudodifferential equations, Lecture Notes in Math., vol. 287, Springer, BerlinHeidelberg-New York, 1973.

[14] Tahara, H., Fuchsian type equations and Fuchsian hyperbolic equations, Japan. J. Math. 5 (1979), 245-347.

[15] Taniguchi, K. and Y. Tozaki, A hyperbolic equation with double characteristics which has a solution with branching singularities, Math. Japon. 25 (1980), 279-300.

[16] Uchikoshi, K., Microdifferential equations with non-involutory characteristics, Comm. Partial Differential Equations 15 (1990), 1-58.

[17] Uchikoshi, K., Microdifferential equations with non-involutory factors, Comm. Partial Differential Equations 18 (1993), 1557-1587.

[18] Yamane, H., Branching of singularities for some second or third order microhyperbolic operators, J. Math. Sci. Univ. Tokyo. 2 (1995), 671-749.

(Received July 18, 1996)

Department of Mathematics

National Defense Academy

Hashirimizu 1-10-20

Yokosuka, Japan

E-mail: uchikosh@cc.nda.ac.jp 